\title{
Was bedeutet das für meinen \\ Landkreis oder meine Gemeinde? - Zu den Auswirkungen von Multilokalität in ländlichen Räumen
}

\author{
Lena Greinke, Martin Albrecht, Frank Othengrafen, \\ Jens-Martin Gutsche und Franziska Lehmann
}

\subsection{Auswirkungen von Multilokalität in ländlichen Räumen}

Multilokalität ist von hoher gesellschaftlicher sowie raum- und regionalplanerische Relevanz (Weichhart 2009: 10), was sich unter anderem darin widerspiegelt, dass das Leben an zwei oder mehr Orten zu temporären An- und Abwesenheiten der Individuen führt. Dabei zeigen die Analysen im Landkreis Diepholz, dass Multilokalität kein Phänomen ist, welches ausschließlich

L. Greinke $(\bowtie)$

Institut für Umweltplanung, Leibniz Universität Hannover, Hannover, Deutschland E-Mail: greinke@umwelt.uni-hannover.de

M. Albrecht · J.-M. Gutsche

Gertz Gutsche Rümenapp - Stadtentwicklung und Mobilität, Hamburg, Deutschland E-Mail: albrecht@ggr-planung.de

J.-M. Gutsche

E-Mail: gutsche@ggr-planung.de

F. Othengrafen

Fakultät Raumplanung, Fachgebiet Stadt- und Regionalplanung, Technische

Universität Dortmund, Dortmund, Deutschland

E-Mail: frank.othengrafen@tu-dortmund.de

F. Lehmann

proloco - Bremen, Bremen, Deutschland

E-Mail: lehmann@proloco-bremen.de

(C) Der/die Autor(en) 2021

F. Othengrafen et al. (Hrsg.), Temporäre An- und Abwesenheiten in ländlichen

Räumen, https://doi.org/10.1007/978-3-658-32500-8_5 
in urbanen Räumen, sondern auch in ländlichen Räumen stattfindet (vgl. Beiträge Greinke/Lange/Born (Kap. 2) und Greinke/Lange (Kap. 4) in diesem Band). Besonders im ländlich geprägten südlichen Teil des Landkreises Diepholz sind verschiedene Formen multilokaler Lebensweisen vorzufinden. $\mathrm{Zu}$ den Incomings gehören z. B. Studierende, die aufgrund des dualen Studiums in unregelmäßigen Zeiträumen im Landkreis wohnen. Außerdem sind einige Kommunen geprägt von landwirtschaftlicher Produktion verschiedener Feldfrüchte, die im Laufe des Jahres zumeist von multilokal Lebenden aus Osteuropa geerntet werden. Zudem gibt es Arbeitnehmende, die phasenweise berufsbedingt aufgrund der dort ansässigen Unternehmen in den Landkreis Diepholz kommen. Darüber hinaus gibt es in einigen Kommunen saisonal oder phasenweise anwesende Dauercamper*innen und Ferienhausbesitzer*innen. Diese reisen z. B. an den Wochenenden oder in den Sommermonaten in den Landkreis und sind in den Wintermonaten abwesend (und an ihren anderen Wohnorten temporär anwesend). Die Outgoings hingegen verlassen die Kommunen im Landkreis Diepholz regelmäßig. Dazu zählen z. B. (Fach-)Arbeitskräfte oder Studierende, die in anderen Kommunen arbeiten oder studieren und deswegen innerhalb der Woche i. d. R. nicht anwesend sind.

Aus diesen phasenweisen An- bzw. Abwesenheiten ergeben sich vielschichtige Konsequenzen für die Orte, z. B. in Hinblick auf den Erhalt, die Bereitstellung sowie Finanzierung von technischer und sozialer Infrastruktur, die Nachfrage auf dem Wohnungsmarkt (Dittrich-Wesbuer et al. 2015) oder die öffentliche Bereitstellung von Mobilitätsangeboten (Weichhart 2009). Darüber hinaus wirkt sich das Führen eines multilokalen Lebensstils auch auf das bürgerschaftliche Engagement der betroffenen Personen sowie den gesellschaftlichen Zusammenhalt aus (Dirksmeier 2010; Lange 2018; Schier 2014).

Ziel des vorliegenden Beitrags ist es, die vielschichtigen, bislang nur unzureichend erforschten Auswirkungen multilokaler Lebensweisen in ländlich geprägten Räumen (vgl. Beitrag Greinke/Lehmann/Othengrafen/Seitz in diesem Band (Kap. 7)) in den Bereichen (1) Wohnen und Wohnungsmarkt, (2) bürgerschaftliches Engagement, (3) kommunale Finanzen und (4) Infrastrukturen zu analysieren. Hierbei stehen v. a. folgende Fragen im Vordergrund: Welche Auswirkungen entstehen durch die diversen multilokalen Lebensweisen? Wie wirken sich die Ansprüche und Bedürfnisse der Multilokalen in den ausgewählten Themenfeldern aus? Inwiefern sind multilokale Lebensweisen nachhaltig (oder nicht)? Dafür werden auf Basis der empirischen Untersuchungen (vgl. v. a. Beitrag Greinke/Lange in diesem Band (Kap.4)) zunächst die Auswirkungen multilokaler Lebensweisen auf den Wohnungsmarkt und das bürgerschaftliche Engagement diskutiert. Danach stehen die Auswirkungen von Multilokalität auf soziale und technische Infrastrukturen sowie auf kommunale Finanzen im Vordergrund; die Betrachtungen dazu beruhen auf theoretischen Überlegungen und 
eigenen Berechnungen. Abschließend werden die Auswirkungen multilokaler Lebensweisen vor dem Hintergrund einer nachhaltigen Entwicklung zusammenfassend untersucht.

\subsubsection{Multilokalität und Wohnen}

Multilokale Lebensweisen können sich auf den Wohnungsmarkt und die Wohnraumentwicklung auswirken (siehe Abb. 5.3). Die empirischen Ergebnisse der Haushaltsbefragung im Landkreis Diepholz machen deutlich, dass $39 \%$ der befragten Multilokalen in einer Zweitwohnung leben, $12 \%$ ein Hotelzimmer und $11 \%$ die Unterkunft eines Partners/einer Partnerin nutzen. Darüber hinaus wohnen $7 \%$ in Ferienwohnungen und $5 \%$ in einem Wohnwagen (siehe Abb. 5.1).

Die qualitativen Interviews mit den Multilokalen zeigen zudem, dass Multilokale spezifische Bedürfnisse an ihren Wohnraum haben. Berufs- und ausbildungsbedingte Incomings bevorzugen bspw. kleine und oftmals möblierte Wohnungen (1-2 Zimmer) in unmittelbarer Nähe zur Arbeitsstelle oder dem Ausbildungs- beziehungsweise Studienort. Im Landkreis Diepholz gibt es bereits privatwirtschaftliche Akteur*innen, die mit der Einrichtung von Boardinghäusern auf diese Nachfrage reagiert haben. Da jedoch der Wohnungsbestand in Niedersachsen, speziell im Landkreis Diepholz, insgesamt sehr homogen von Ein- und Zweifamilienhäusern geprägt ist (NBank 2017: 17; InWIS 2016: 68), fehlen passende Wohnraumangebote für Multilokale. Die Eigenheimquote des Landkreises Diepholz liegt bei $77 \%$ und damit über dem Landesdurchschnitt von ca. 60 \% (NBank 2017: 106). Die erhöhte Wohnraumnachfrage spüren besonders die Stadt Diepholz und die Samtgemeinde Altes Amt Lemförde (InWIS 2016).

Gleichzeitig wird die Wohnraumsuche für Multilokale durch den schwach ausgeprägten Mietwohnungsmarkt im Landkreis Diepholz erschwert. Das wird in der recht geringen Anzahl fertiggestellter Wohnungen deutlich. In 2017 wurden 375 Wohnungen in neuen Ein- und Zwei-Familienhäusern und 252 Wohnungen in neuen Mehrfamilienhäusern fertiggestellt. Außerdem wurde in 2016 und 2017 insgesamt lediglich 47,1 ha Wohnbauland (703 Wohneinheiten) neu ausgewiesen (NBank 2019: 74, 76). Die vorhandenen Mietwohnungen verfügen zudem in der Regel über 2-3 Zimmer mit einer Wohnfläche von ca. 70-80 $\mathrm{m}^{2}$, welche aufgrund der Größe für Incomings und Einpersonenhaushalte ungeeignet sind. Besonders in der Kreisstadt Diepholz, der Samtgemeinde Altes Amt Lemförde und in der Samtgemeinde Barnstorf besteht ein Bedarf an kleinen Single- und Paarwohnungen (InWIS 2016: 21, 26). Der Anteil von Klein- bzw. Kleinstwohnungen ist in diesen Kommunen im Vergleich zu anderen Teilräumen bereits vergleichsweise 


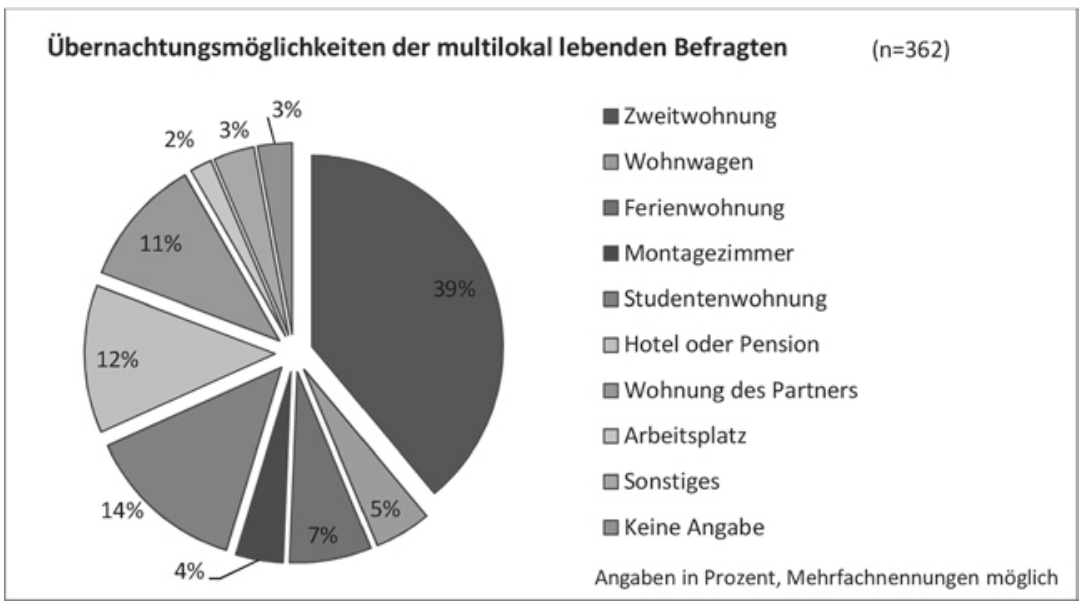

Abb. 5.1 Übernachtungsmöglichkeiten der multilokal lebenden Befragten im Landkreis Diepholz (Quelle: TempALand 2020)

hoch (ca. $7 \%$ des gesamten Wohnungsbestandes in der Samtgemeinde Altes Amt Lemförde), aber mittel- und langfristig noch nicht ausreichend (InWIS 2016: 26), weil davon auszugehen ist, dass die bisherige Nachfrage noch weiter steigt.

Die Nachfrage nach 1-2 Zimmer-Wohnungen erhöht sich zudem durch unterschiedliche Nutzer*innengruppen in einigen Teilräumen deutlich, z. B. in der Stadt Syke, der Gemeinde Weyhe und der Stadt Sulingen (siehe Abb. 5.2). Nicht nur (Fach-)Arbeitskräfte, die im Diepholzer Land bei dort ansässigen (international tätigen) Großunternehmen beschäftigt sind (InWIS 2016: 26), sondern auch die Kreisverwaltung, der Bundeswehrstandort und die Private Hochschule für Wirtschaft und Technik (phwt) in der Stadt Diepholz erhöhen die Wohnraumnachfrage insbesondere in diesem Segment. Darüber hinaus verstärken die zunehmende Alterung der Bevölkerung und allgemeine Singularisierungstrends den Anstieg der Nachfrage. Dementsprechend sind es nicht nur Multilokale, (Fach-)Arbeitskräfte, Auszubildende und Studierende, sondern auch Singles, Geringverdienende, Erwerbslose und Senior*innen, die bezahlbare 1-2 Zimmer Wohnungen nachfragen (NBank 2017: 69). Bislang konzentriert sich die kommunale Wohnungsbaugesellschaft im Landkreis Diepholz noch nicht auf den Bau kleinerer Wohnungen; allerdings setzt sie derzeit ein Pilotprojekt zum Bau von sogenannten Variowohnungen um, die durch modulare Wohneinheiten für verschiedene Zielgruppen angepasst werden können. 


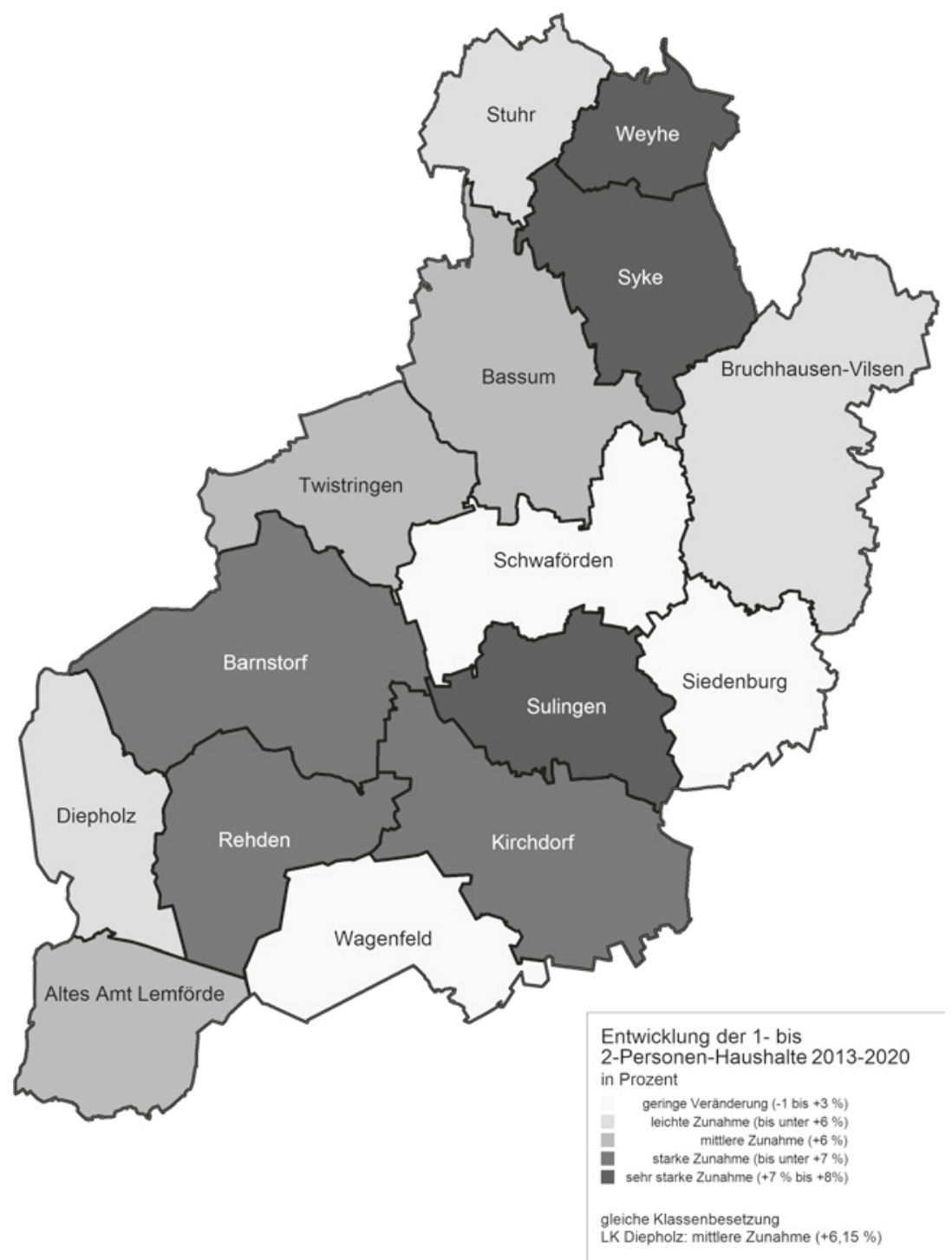

Abb. 5.2 Entwicklung der Anzahl der 1- bis 2-Personen-Haushalte in den Kommunen im Landkreis Diepholz, 2013-2020 (Quelle: InWIS 2016: 42) 


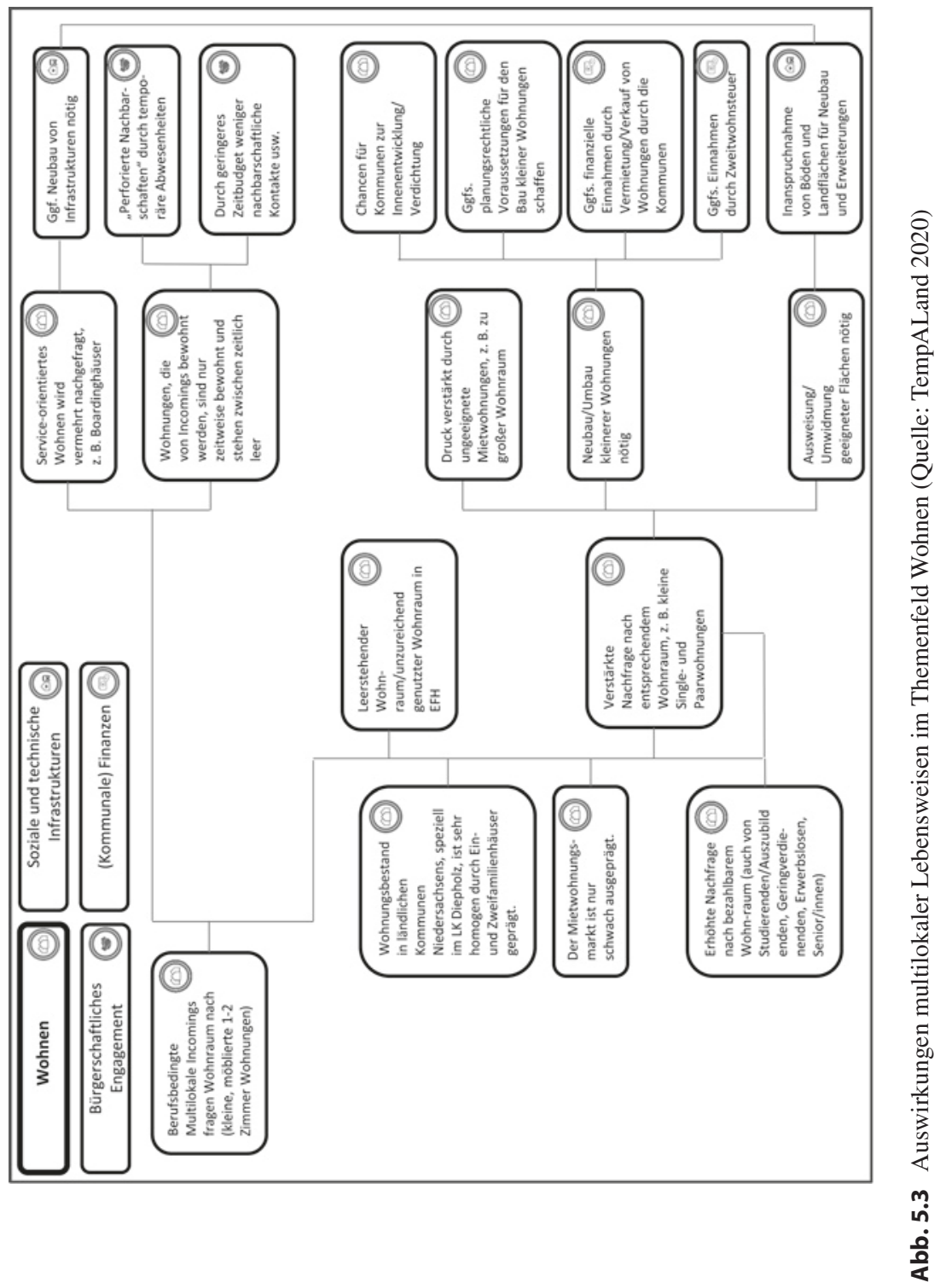


Folglich wird wie im Landkreis Diepholz der Umbau von zu großem Wohnraum in kleinere Wohneinheiten oder der Neubau kleiner Wohnungen nötig. Dafür ist vielerorts die Umwidmung geeigneter Flächen oder eine Neuausweisung von Baugebieten für den Geschosswohnungsbau erforderlich. Besonders in ländlichen Räumen fehlen geeignete und entsprechend ausgewiesene Flächen oft. Überdies müssen für den Bau kleiner Wohnungen ggf. planungsrechtliche Vorausetzungen geschaffen werden. Eine Neuausweisung von Flächen kann zu einer erhöhten Inanspruchnahme von Boden und Landfläche führen und mit Zielen der nachhaltigen Entwicklung kollidieren. Zudem können daraus Kosten für Kommunen durch den Neubau von Infrastrukturen entstehen, die bereitgestellt werden müssen. Dementsprechend sollten zunächst ausgewiesene Flächenreserven umgewidmet werden, bevor neue Flächen in Anspruch genommen werden. Ein bedarfsgerechter Bau kleinerer Wohnungen kann jedoch vor dem Hintergrund einer nachhaltigen Entwicklung immer noch vorteilhafter sein, als die Realisierung von Einfamilienhäusern, die deutlich mehr Fläche pro Person in Anspruch nehmen.

Der Druck auf den Wohnungsmarkt kann für die Kommunen aber auch eine Chance sein, die Innenentwicklung oder Verdichtung in den Regionen zu fokussieren. Mit Hilfe von Baulandausweisungen nahezu ausschließlich im Innenbereich und auf Brachflächen, z. B. Bauen in zweiter Reihe, sowie der Umnutzung oder des Umbaus landwirtschaftlich geprägter (Wohn-)Gebäude im öffentlichen Raum können negativen Folgen, wie bspw. zersiedelten Ortschaften, vorgebeugt werden. Gleichzeitig können Kommunen auch geringfügig finanziell vom Neu- und Umbau profitieren (z. B. durch erhöhte Steuereinnahmen). Überdies können Kommunen eine Zweitwohnsitzsteuer auf Nebenwohnungen erheben und dadurch möglicherweise finanziell profitieren. Empirische Erhebungen zur Budgetkonsolidierung für Kommunen gibt es bislang noch nicht. In Österreich hat sich jedoch gezeigt, dass die Zweitwohnungssteuer in finanzschwachen, touristisch geprägten Kommunen durchaus zu erhöhten Steuereinnahmen der Kommunen beitragen kann (Dittrich-Wesbuer/Sturm 2020).

Die Ein- und Zwei-Familienhäuser werden aufgrund der alternden Bevölkerung darüber hinaus oftmals unzureichend genutzt (Ein-Personen-Haushalte) oder stehen sogar leer. Im Jahr 2018 betrug der Wohnungsleerstand im Landkreis Diepholz laut LSN (2019) 3,2 \%, was unter dem niedersächsischen Durchschnitt von 3,7\% liegt (Zensusdatenbank 2019). Hier können multilokale Lebensweisen eine Chance für ländliche Räume darstellen, denn phasenweise zuziehende Menschen können bspw. leerstehende oder nicht ganzjährig genutzte Gebäude bewohnen und somit zu einer zeitweisen Belebung und auch besseren Auslastung der Infrastruktur beitragen. In einigen Kommunen des 
Diepholzer Landes, z. B. der Gemeinde Wagenfeld, der Samtgemeinde Altes Amt Lemförde und der Stadt Diepholz, werden solche Leerstände bereits zeitweise durch saisonale Arbeitskräfte genutzt. Außerdem weichen einige Incomings, v. a. Studierende, auf leerstehende Ferienhäuser als temporäre Wohnsitze aus. Für Immobilienbesitzer*innen besteht somit die Chance, Wohnraum zumindest zeitweise zu vermieten oder zu groß gewordenen Wohnraum umzubauen.

Anders als Incomings verursachen Outgoings kaum Folgen am Wohnungsmarkt: In Mehrpersonenhaushalten ist generell nicht mit einer Veränderung der Wohnsituation durch Outgoings zu rechnen, weil sie keinen zusätzlichen Wohnraum im Landkreis nachfragen und der bestehende Wohnraum von der Familie oder dem/der Partner*in weitergenutzt wird („Einfamilienhaus am Hauptwohnort bleibt unverändert erhalten"). Somit steht dadurch für Incomings kein zusätzlicher Wohnraum zur Verfügung. Der Wohnraum allein lebender Outgoings könnte theoretisch zwar phasenweise genutzt werden, z. B. in der Woche (bei überwiegend arbeitsbedingten Outgoings) oder auch am Wochenende (bei eher privat bedingten Outgoings), allerdings zeigen die Befragungen im Landkreis Diepholz nur eine geringe Bereitschaft von Outgoings, ihren phasenweise leerstehenden Wohnraum in der Zeit der Abwesenheit unterzuvermieten.

Die phasenweisen An- und Abwesenheiten in ländlichen Räumen können besonders auch in Gebieten mit langjährig gewachsenen Nachbarschaften für Konflikte sorgen. Untersuchungen in touristisch geprägten, strukturstarken ländlichen Räumen haben gezeigt, dass die Abwesenheiten von Multilokalen für Konfliktpotenzial zwischen der multi- und monolokalen Bevölkerung sorgen können (z. B. durch heruntergelassene Rollläden und ungepflegte Vorgärten) (Dirksmeier 2010: 68). In strukturschwächeren ländlichen Regionen, in denen Nachbarschaften sowieso schon durch Leerstände „perforiert“ werden, kann die phasenweise Abwesenheit multilokal Lebender die negativen Folgen, z. B. unbelebte Quartiere, Viertel oder Dörfer, verstärken. Im Landkreis Diepholz sind derartige Entwicklungen (noch) nicht zu beobachten; hier hält sich der Leerstand aufgrund der räumlichen Nähe zu Bremen und der, im Vergleich zu anderen ländlichen Räumen, eher positiven wirtschaftlichen Entwicklung (vgl. Beitrag Greinke/Lehmann/Othengrafen/Seitz in diesem Band (Kap. 7)) in Grenzen. Aber in eher peripheren ländlichen Räumen können Leerstände und die geringen Zeitbudgets der Multilokalen, die aufgrund der häufigen Ortswechsel oder starken beruflichen und privaten Verpflichtungen entstehen, zu einer Gefährdung des sozialen Zusammenhalts beitragen.

Der Wohnungsmarkt und die Entwicklung des Wohnraums werden folglich unmittelbar durch multilokale Lebensweisen beeinflusst (vgl. Abb. 5.3). Neben 
einer erhöhten Nachfrage nach kleinen Wohnungen können mehrörtige Lebensweisen in betroffenen Kommunen auch dazu beitragen, Wohnraum um- oder nachzunutzen und damit auch die Innenentwicklung in Kommunen im ländlichen Raum stärken. Neben geringen Konsolidierungen aus Steuereinnahmen lässt sich ggf. aber auch Konfliktpotenzial erwarten. Besonders in ländlichen Räumen können „perforierte Nachbarschaften“ Herausforderungen darstellen, mit denen die Kommunen umgehen müssen (vgl. Beitrag Greinke/Lehmann/Othengrafen/ Seitz in diesem Band (Kap. 7)).

\subsubsection{Multilokalität und bürgerschaftliches Engagement}

Neben der veränderten Nachfrage auf dem Wohnungsmarkt wirken sich multilokale Lebensweisen auf das bürgerschaftliche Engagement der mehrörtig lebenden Personen aus (vgl. Lange 2018). Unter bürgerschaftlichem Engagement wird in diesem Beitrag die Gesamtheit von freiwilligen, gemeinnützigen und unbezahlten Tätigkeiten verstanden (Kahle/Schäfer 2005), die zugleich das klassische Ehrenamt, d. h. die Übernahme von formell definierten Ämtern und Funktionen im Rahmen von Vereinigungen und Institutionen, umfasst (Becker et al. 2010). Demnach ist „bürgerschaftliches Engagement freiwillig, nicht auf materiellen Gewinn gerichtet, gemeinwohlorientiert, öffentlich beziehungsweise findet im öffentlichen Raum statt, [und] wird in der Regel gemeinschaftlich beziehungsweise kooperativ ausgeübt" (Deutscher Bundestag 2002: 38 f.). Neben Baden-Württemberg und RheinlandPfalz ist das bürgerschaftliche Engagement in Niedersachsen überdurchschnittlich ausgeprägt, dies gilt insbesondere für die eher ländlich geprägten Teilräume. Dabei wird deutlich, dass mit steigender Größe der Gemeinde das bürgerschaftliche Engagement abnimmt und insbesondere kleinere Gemeinden den höchsten Anteil an Engagierten aufweisen (s.a. Prognos AG et al. 2008: 17 ff.).

Dem bürgerschaftlichen Engagement kommt vor allem unter veränderten demografischen Bedingungen eine besondere Rolle zu (u. a. Bieker et al. 2004; Voß et al. 2011; BMFSFJ 2016). Durch den agrarstrukturellen und demografischen Wandel sowie die Finanzknappheit der öffentlichen Hand sind ländliche Räume in ihrer Entwicklung oftmals stark beeinträchtigt. Vielfach stehen ländliche Räume und ein Großteil der Dörfer vor mannigfaltigen Problemen und müssen neben Arbeitsplatz- und Bevölkerungsrückgängen sowie Gebäudeleerständen und -verfall, auch Attraktivitätsminderungen des Orts- und Landschaftsbilds und weitere Infrastrukturverluste bewältigen (Siedentop et al. 2011; Voß et al. 2011). Hier scheint eine Neuinterpretation des raumordnerischen Ziels der gleichwertigen Lebensverhältnisse erforderlich zu sein, bei der ländlichen Räumen eine stärkere Selbstver- 
antwortung zukommt (Blotevogel/Danielzyk 2006; Aring 2010). Zukünftig werden demnach flexiblere, stärker privatwirtschaftlich oder bürgerschaftlich organisierte Infrastrukturen und neue Formen der Steuerung und Mitwirkung benötigt. Um die Zukunftsfähigkeit von Dörfern langfristig und nachhaltig sicherzustellen, sind bereits viele Kommunen aktiv geworden, in dem sie ihre Ortskerne revitalisieren und sich um eine stabile lokale Ökonomie sowie die Sicherung, Vorhaltung und den Ausbau von Infrastrukturen bemühen. Darüber hinaus sind sie bestrebt, die Aktivitäten dörflicher Vereine zu sichern und zu fördern, bürgerschaftliches Engagement und Verantwortungsgefühl zu stärken (Kötter 2009; Henkel 2010). Dazu sind allerdings funktionsfähige, lebendige Gemeinschaften in den Dörfern und ein verändertes Staatsverständnis hin zu bürgerschaftlichem bzw. freiwilligem Engagement entscheidend (Olk/Hartnuß 2011). Die nachfolgenden Ausführungen zeigen, inwiefern temporäre An- und Abwesenheiten bürgerschaftliches Engagement beeinflussen.

In Bezug auf das bürgerschaftliche Engagement in ländlichen Räumen können multilokal lebende Personen sowohl Chance als auch Herausforderung sein (siehe Abb. 5.4). Die Interviews mit den Multilokalen im Landkreis Diepholz sowie andere Untersuchungen (z. B. Lange 2018) haben gezeigt, dass das bürgerschaftliche Engagement der Multilokalen sehr divers ist und stark von den Motiven und der „Wanderungsrichtung“ abhängt. Auf der einen Seite wird deutlich, dass Incomings sich aufgrund hoher Arbeitsbelastung und geringer Zeitverfügbarkeit nur im Einzelfall am Nebenort im Landkreis Diepholz engagieren. Auf der anderen Seite ist für einige Incomings aber auch das Angebot an Engagementmöglichkeiten inhaltlich nicht interessant genug und nicht passend zu ihren zeitlichen Möglichkeiten, sodass sie keine geeigneten Offerten finden und somit auch nicht für freiwillige Tätigkeiten gewonnen werden können. Darüber hinaus gibt es auch unter den Multilokalen Personen, die kein Interesse am bürgerschaftlichen Engagement haben, weder am Haupt- noch am Nebenort (Lange 2018).

Überdies kommen aber auch Incomings in das Diepholzer Land, die explizit an einem Engagement interessiert sind, um im Landkreis ,anzukommen“ und Kontakte zu knüpfen. Viele Multilokale beklagten in den Interviews, dass sie nach eigenen Recherchen zwar freiwillige Tätigkeiten gefunden haben, allerdings hätten sie sich generell mehr Informationen zu den Angeboten im Landkreis Diepholz gewünscht. Demgegenüber finden laut Aussage der Bürgermeister und Akteur*innen aus den Vereinen viele Bewohner*innen und Zuziehende des Landkreises Diepholz den Weg zum Engagement selbstständig. Besonders über die Kinder- und Jugendarbeit können sich neuzugezogene Familien schnell einbringen und knüpfen Kontakte zu anderen Eltern und Akteur*innen aus Vereinen oder dem Ort. Dies ist jedoch für Incomings deutlich schwerer, da sie nicht über diese 


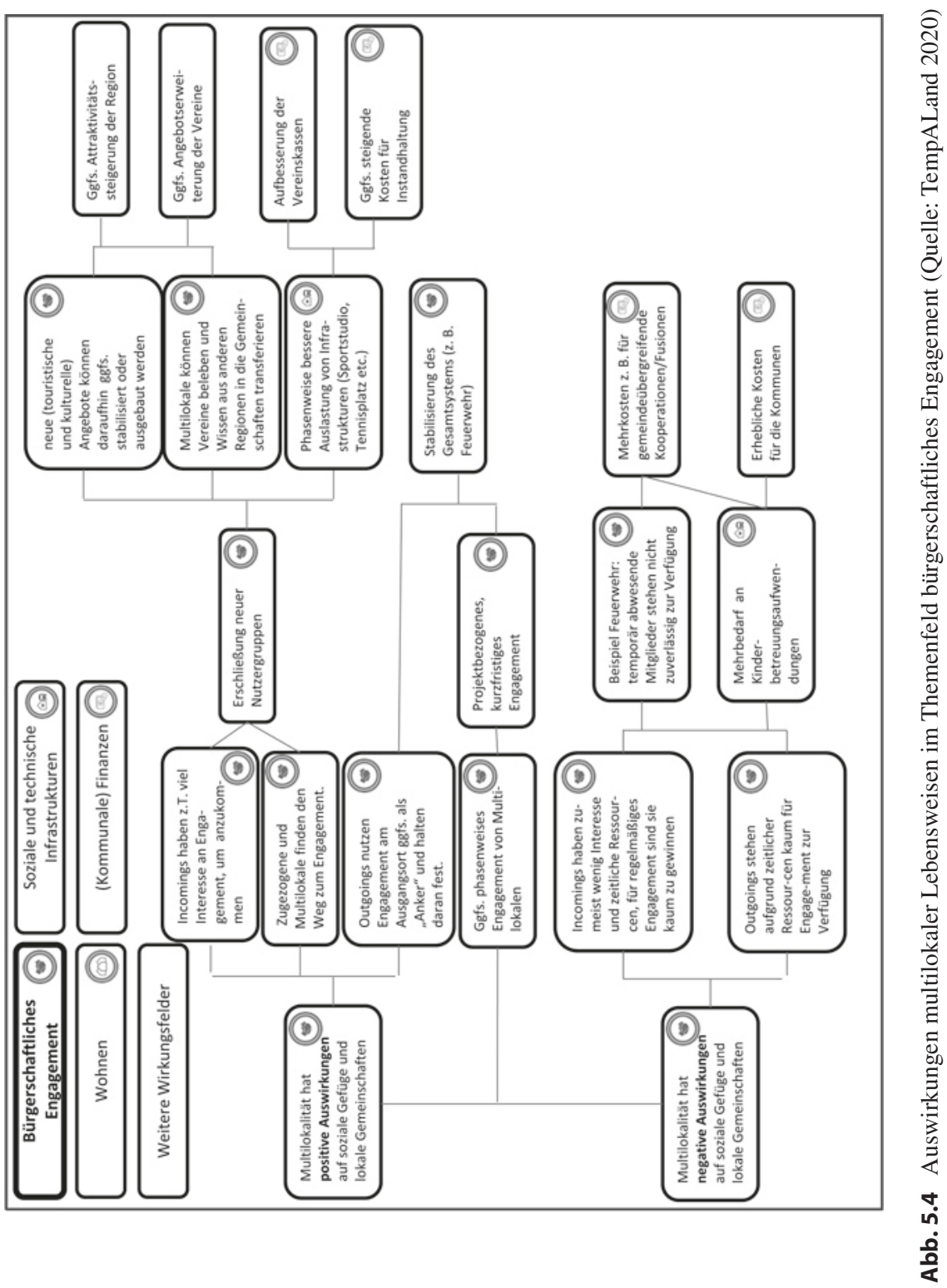


sozialen Netze verfügen und die Informationen über entsprechende Angebote selbst suchen müssen.

Die Interviews mit multilokalen Incomings haben gezeigt, dass einige zum Beispiel an sportlichen Aktivitäten im Landkreis Diepholz interessiert sind und sich bei Dienstleistern oder in Vereinen anmelden, um dort ihrem Hobby nachzugehen. Dadurch können Zuziehende und Incomings dafür sorgen, dass Infrastrukturen, wie beispielsweise Sportplätze, zumindest phasenweise besser ausgelastet werden. Durch die vermehrte Nutzung der Angebote und die zusätzlichen Nutzer*innen können z. B. Mitgliederzahlen in Fitnessstudios steigen oder (Sport-)Plätze belebt werden. Das wiederum kann dabei helfen, angespannte Vereinskassen aufzubessern: Denn wenn die Multilokalen und Zuziehenden als neue Mitglieder gewonnen werden können oder zumindest phasenweise das Angebot nutzen, müssen sie in der Regel dafür einen Mitgliedsbeitrag zahlen, den die Vereine in die Erhaltung ihrer Angebote und Infrastrukturen einbringen können. Diese Einnahmen können Vereine zudem auch in die Schaffung neuer Angebote investieren und somit die Attraktivität der Region und der Kommunen erhöhen. Allerdings können durch die höheren Nutzungsfrequenzen auch steigende Kosten für die Instandhaltung der Infrastrukturen entstehen, die die Betreibenden ggf. nicht nur über die Einnahmen abfedern können und somit vor der Herausforderung stehen, die Infrastrukturen angemessen aufrecht zu erhalten.

Des Weiteren können Incomings eine Chance für Kommunen sein, indem sie touristische und kulturelle Angebote nutzen und diese dadurch stabilisieren. Im Landkreis Diepholz stehen die Anbieter*innen noch nicht vor der Herausforderung, dass sie ihre Angebote nicht aufrechterhalten können, allerdings gibt es ländliche Kommunen, die auf diese Entwicklungen reagieren müssen. Besonders in peripheren ländlichen Räumen werden einige Leistungen der Daseinsvorsorge (z. B. Verkehrsinfrastrukturen, Schwimmbäder, Bibliotheken etc.) zivilgesellschaftlich und über das Engagement der Bevölkerung vor Ort bereitgestellt (vgl. Steinführer 2015). Multilokale könnten hier zumindest teilweise zur Stabilisierung der Mitgliederzahlen beitragen und sich phasenweise (in Vereinen) engagieren.

Darüber hinaus ist vielerorts die Gefahrenabwehr durch die Feuerwehren zivilgesellschaftlich organisiert und wird durch freiwilliges Engagement aufrechterhalten. Die Personen in der Feuerwehr sind zumeist nicht hauptberuflich dort tätig, sondern engagieren sich freiwillig, um die Sicherheit in den Orten zu gewährleisten. Hier ist denkbar, dass in Kommunen, in denen aufgrund der fehlenden Einsatzstärke der Feuerwehr die Sicherung der Gefahrenabwehr gefährdet ist, Incomings über eine bereits in einigen Kommunen etablierte 
Doppelmitgliedschaft zumindest temporär die Einsatzstärke erhöhen können. Die Untersuchungen haben allerdings gezeigt, dass Multilokale im Landkreis Diepholz bislang (noch) nicht in Form von Doppelmitgliedschaften in den Feuerwehren der Kommunen tätig sind.

Ein Wegfall der beispielhaft beschriebenen Daseinsvorsorgeleistungen kann sich nicht nur auf die Attraktivität der Ortschaften, sondern auch unmittelbar auf die Gefahrenabwehr und somit die Sicherheit vor Ort auswirken. Sollten diese Leistungen nicht mehr über die Zivilgesellschaft abgedeckt werden können, kann dies schwerwiegende Folgen für die Orte haben. Daraus kann eine Abwärtsspirale entstehen, in der sich die negativen Auswirkungen vor Ort verstärken und bis hin zur Entleerung ganzer Dörfer führen können, auch weil die Sicherheit und der soziale Zusammenhalt nicht mehr gewährleistet werden können. Besonders Outgoings setzen oft ihre Prioritäten anders und versuchen, ihre frei verfügbare Zeit mit ihrer Familie oder Freund*innen zu verbringen, so dass für freiwilliges Engagement keine Zeit mehr bleibt. Sollten deshalb Feuerwehren, z. B. aufgrund fehlender Mitglieder durch alternde Bevölkerung oder Pendler*innen und Multilokale, nicht mehr aufrechterhalten werden können, kann dies zusätzlichen finanziellen und planerischen Aufwand bedeuten, weil Kommunen gemeindeübergreifende Kooperationen oder Fusionen organisieren und umsetzen müssen (vgl. Kap. 5.1.3).

Andererseits sind Outgoings in Teilen stark engagiert an ihren Orten. Dies betrifft vor allem freizeitbedingt multilokal Lebende, die zumeist beruflich nicht so stark eingespannt sind oder sich bereits im Ruhestand befinden und somit die zeitlichen Ressourcen haben, sich zu engagieren. Im Landkreis Diepholz gibt es zum Beispiel multilokal lebende Paare im Ruhestand, die ganz explizit ihre Lebensweise an ihre Termine anpassen. So bleiben sie z. B. auch mal länger an ihrem Wohnort im Landkreis Diepholz, weil sie dort engagiert sind und bspw. bei der Vorbereitung einer Veranstaltung o.ä. helfen wollen. Andererseits haben sie auch an ihrem anderen Ort „Engagementverpflichtungen“, denen sie regelmäßig nachgehen; damit fehlen sie an beiden Orten zeitweise, engagieren sich dafür während ihrer Anwesenheit sehr stark. Weiterhin gibt es Outgoings, die ein Engagement als "Anker" am Ausgangsort nutzen und deshalb an ihren Aktivitäten (z. B. in Sportvereinen), wenn auch zeitlich eingeschränkt, weiterhin festhalten. Über diese Verbindung gelingt es den Outgoings, trotz ihrer geringen Anwesenheitszeiten Kontakt zur Gemeinschaft zu halten. Die Interviews im Landkreis Diepholz haben zusätzlich gezeigt, dass es sogar Multilokale gibt, die ihren Aufenthaltsrhythmus unterbrechen bzw. spontan zirkulieren, um ihre Sportmannschaft in einem Spiel zu unterstützen. Da allerdings insbesondere den arbeitsbedingten Outgoings die zeitlichen Ressourcen für ein Engagement fehlen, 
bevorzugen sie oft ein projektbezogenes oder kurzfristiges Engagement, welches sie nur phasenweise ausüben können. Das kann dazu beitragen, dass das Gesamtsystem in Vereinen und Institutionen der Regionen und Kommunen stabilisiert wird. Wenn auch nur zeit- oder phasenweise, stehen die Outgoings immerhin in ihren Zeiten der Anwesenheit zur Verfügung.

Im Bereich des bürgerschaftlichen Engagements wird deutlich, dass das Angebot und die Aktivitäten in ländlichen Räumen von besonderer Bedeutung sind, nicht nur für die Sicherung der Daseinsvorsorgeleistungen, sondern auch für den gesellschaftlichen Zusammenhalt (vgl. auch Abb. 5.5). Vielerorts müssen die Bürger*innen bereits wichtige Aufgaben der Daseinsvorsorge übernehmen, um Infrastrukturen und Angebote aufrecht zu erhalten. Eine zukünftige Herausforderung ist es deshalb, multilokal lebende Personen in lokale Gemeinschaften zu integrieren und ihnen den Weg zum Engagement zu ermöglichen. Dafür ist es unerlässlich, Angebotsformen und -formate stärker auf die geringen Zeitressourcen der Multilokalen, Pendler*innen und anderer Zielgruppen zuzuschneiden und passende Aktivitäten zu entwickeln. Dies geht einher mit generellen Veränderungen beim bürgerschaftlichen Engagement; auch hier ist zu beobachten, dass sich Nachfragestrukturen nach ehrenamtlichen Tätigkeiten ändern und kurzfristiger, projektbezogener und flexibler werden (müssen) (vgl. Lange 2018), wodurch Anpassungen auf der Angebotsseite unabdingbar werden.

Multilokal lebende Personen können in ländlichen Räumen einen Beitrag leisten, nicht nur für Engagementtätigkeiten, sondern auch besonders für den Erhalt und die Bereitstellung von Daseinsvorsorgeleistungen. Allerdings dürfen Infrastrukturen nicht nur privatwirtschaftlich oder bürgerschaftlich organisiert sein, sondern müssen weiterhin auch staatliche Aufgabe bleiben, um Strukturen in den Orten aufrecht zu erhalten und dauerhaft zu sichern. Die Verantwortung darf folglich nicht ausschließlich auf die Zivilgesellschaft übertragen werden, sondern muss auch weiterhin vom Staat bzw. den Kommunen übernommen werden, um die Attraktivität der Kommunen als Wohn- und Lebensort zu erhalten und zu erhöhen sowie vielerorts die Folgen des demografischen Wandels zu reduzieren.

\subsubsection{Multilokalität und Infrastrukturen, Versorgungsleistungen und kommunale Finanzen}

Multilokal lebende Menschen nutzen grundsätzlich Infrastruktur- und Versorgungsangebote an all ihren Wohn- und Lebensorten. Dabei gilt in Bezug auf die wohnungsbezogenen Infrastrukturen, dass das Leben an mehreren Wohnorten im 
Vergleich zum Leben an einem Wohnort mit einer Mehr-Nutzung von Infrastruktur verbunden ist: So sind z. B. alle genutzten Wohnungen in aller Regel an die entsprechenden Netze (Strom, Wasserver- und -entsorgung, ggf. Gas) angeschlossen und werden regelmäßig von der Müllabfuhr bedient, auch wenn Entnahme- oder Einleitungsmengen im Vergleich zu dauerhaft genutzten Wohnungen ggf. geringer sind bzw. nur zeitweise auftreten. Beispielsweise im Bereich des abwehrenden Brandschutzes oder des Rettungswesens werden Wohnungen in die Bedarfsplanung einbezogen, auch wenn diese nur temporär genutzt werden. Dies ist für die Leistungserbringer*innen bei einzelnen nur zeitweise genutzten Wohnungen in integrierten Lagen vergleichsweise unproblematisch. Es sind jedoch auch Fallbeispiele z. B. in touristisch besonders attraktiven Gebieten bekannt, bei denen eine Vielzahl von Zweitwohnsitzen in Verbindung mit einer „klassischen“ touristischen Nutzung einen Einfluss auf die Vorhaltung und den Betrieb von Rettungsmitteln haben (z. B. zusätzliche Vorhaltung von Rettungsmitteln in den Sommermonaten oder eine auch Wochenendhausgebiete berücksichtigende Standortplanung im Bereich der Notfallrettung und Hilfeleistung) (siehe Abb. 5.5).

Darüber hinaus ergibt sich aus einem Leben an mehreren Orten ggf. die Mehrnutzung von Verbindungsinfrastrukturen zwischen den Wohnorten (z. B. Straßeninfrastruktur oder Nutzung von Angeboten des ÖPNV), die gegenüber einem Leben an nur einem Ort grundsätzlich höher ist. Diese kann jedoch dann, wenn z. B. eine Zweitwohnung in der Nähe des Arbeitsortes gewählt wird (und regelmäßige weite Pendelwege vermieden werden), auch geringer sein, als im Falle der Entscheidung für ,nur“" einen Wohnort.

Für die meisten der Versorgungsinfrastrukturen gilt jedoch, dass sich durch ein Leben an mehreren Wohnorten in Summe nicht unbedingt eine Mehr- oder Mindernutzung ergibt, sondern sich eine in der Menge weitgehend konstante Inanspruchnahme vielmehr auf mehrere Orte verteilt - und damit lokal eine im Vergleich zum Leben an nur einem Ort vermehrte oder verminderte Nutzung auftritt. Dabei ist jedoch zwischen folgenden Aspekten zu differenzieren:

- Im Bereich der - für die Kommunen häufig besonders kostensensiblen Betreuungs- und Bildungsinfrastrukturen (insbesondere Kindertagesbetreuung und Schule) - resultiert aus multilokalen Alltagsarrangements häufig kein verändertes Inanspruchnahmeverhalten: Kinder und Jugendliche nutzen Betreuungs- und Bildungsinfrastrukturen in aller Regel nur an ihrem Hauptwohnort. Auch wenn sie, z. B. infolge der Trennung ihrer Eltern, multilokal leben, erfolgen Betreuung und Beschulung grundsätzlich nur an einem Ort. Der Einfluss multilokaler Alltagsarrangements auf die Erfordernisse zur Bereitstellung von Versorgungsleistungen ist damit gering bis nicht existent. 
- Die Nutzung von kommerziellen Angeboten und Dienstleistungen zur täglichen Versorgung (Einkauf oder personenbezogene Dienstleistungen wie z. B. Friseur) erfolgt in aller Regel grundsätzlich an allen Wohnorten des täglichen Lebens (sofern entsprechende Angebote überhaupt an allen Orten vorhanden sind). Dabei gilt jedoch, dass eine Mehrnutzung an einem der Wohnorte mit einer Mindernutzung am anderen Wohnort einhergeht. Anbieter*innen von Versorgungsleistungen können sich darauf grundsätzlich gut einstellen: Beispiele dafür sind z. B. saisonal veränderte Öffnungszeiten oder sogar Schließzeiten bei Angeboten an besonders stark durch touristische oder Zweitwohnungsnutzungen geprägten Gebieten.

- Ähnliches gilt für Sport-, Kultur- oder Freizeitangebote: So können temporär anwesende Personen an einem Ort zur Tragfähigkeit von Angeboten beitragen (z. B. Bücherei, Volkshochschule, Schwimmbad). Gleichzeitig wird sich daraus am anderen Wohnort - im Vergleich zur „dauerhaften“ Nutzung im Falle eines einzigen Wohnortes - eine geringere Auslastung des vergleichbaren Angebotes ergeben.

- Für den Bereich der ärztlichen und gesundheitlichen Versorgung lässt sich feststellen, dass am „Nebenwohnort“ zwar eine „Ad-Hoc-Inanspruchnahme“ von Leistungen erfolgt (z. B. im Falle akuter Erkrankungen, Beschwerden oder Notfallsituationen), längerfristig planbare und Routinetermine jedoch oftmals am (gefühlten) „Hauptwohnort“ wahrgenommen werden (vgl. Leubert 2013). Dies kann insofern Konsequenzen für die Erbringung von Leistungen entfalten, als sich die ärztliche Bedarfsplanung an der Anzahl von Menschen mit Hauptwohnsitz orientiert und sich damit Versorgungsengpässe in besonders durch eine hohe Anzahl von Nebenwohnungen geprägten Regionen ergeben können.

Während die vorstehend beschriebenen Aspekte die Nachfrage nach Versorgungsleistungen durch Menschen mit multilokalen Alltagsarrangements in den Mittelpunkt stellt, sind jedoch auch Folgewirkungen des Lebens an mehreren Orten für die Bereitstellung von Versorgungsleistungen denkbar (vgl. Abb. 5.6). Dies betrifft insbesondere das ehrenamtliche Engagement im Bereich des abwehrenden Brandschutzes sowie des Katastrophenschutzes (durch die freiwilligen Feuerwehren bzw. ehrenamtliche Strukturen in die in den Katastrophenschutz eingebundenen Hilfsorganisationen) sowie die pflegerische Versorgung von Zugehörigen:

- Auch wenn das für das Projektgebiet im Landkreis Diepholz gültige Brandschutzgesetz des Landes Niedersachsen Doppelmitgliedschaften in freiwilligen Feuerwehren an mehreren Orten (z. B. am Wohn- und am Arbeitsort) 


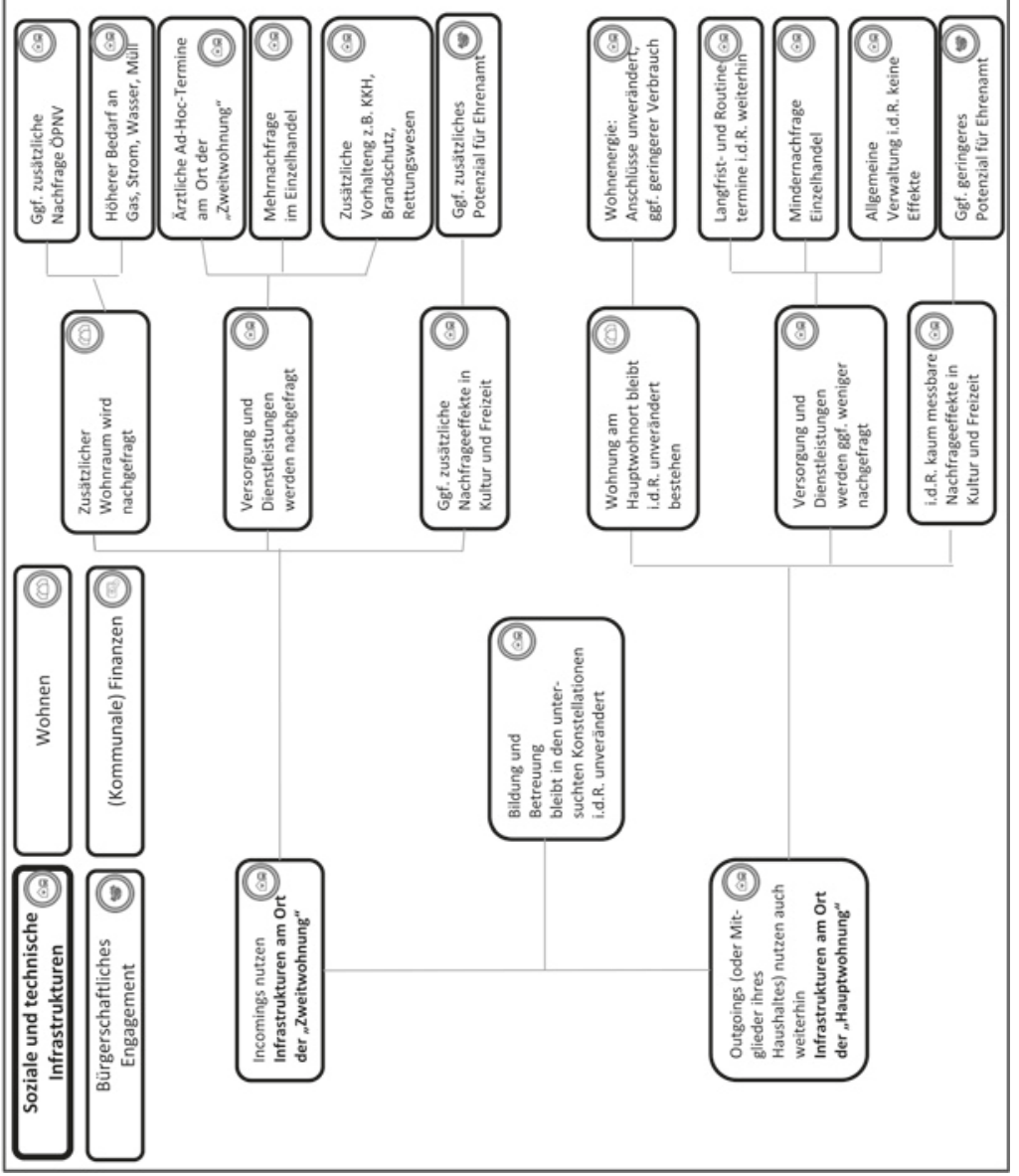

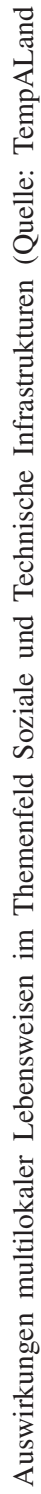

เก

ํํㅇㅇำ 
explizit zulässt, sind Menschen mit mehreren Wohnorten natürlich jeweils nur an einem Ort einsatzbereit. Das bedeutet, dass z. B. Menschen, die beruflich motiviert multilokal leben, zwar unter der Woche am Arbeitsort (=Nebenwohnort) für ein Engagement in der Einsatzabteilung der Freiwilligen Feuerwehr zur Verfügung stehen, gleichzeitig jedoch bei Einsätzen am Hauptwohnort in diesem Zeitraum nicht mitwirken können (und umgekehrt). Dieser Zusammenhang besteht in vielen Gebieten ohne nennenswerten Arbeitsplatzbesatz und einem hohen Anteil auspendelnder Arbeitnehmer*innen grundsätzlich - wird jedoch durch multilokale Alltagsarrangements ggf. verstärkt.

- Im Bereich der pflegerischen Versorgung von Zugehörigen ergibt sich ein zusätzlicher Aspekt: Die Pflege z. B. der eigenen Eltern kann Ausgangspunkt für die Entscheidung für ein multilokales Leben sein ("Ich bin zumindest am Wochenende vor Ort, um die pflegerische Versorgung sicherzustellen"), die damit einhergeht, dass z. B. unter der Woche professionelle Pflegeangebote in Anspruch genommen werden. Daraus kann sich jedoch eine - zumindest gegenüber dem Leben des/der Pflegenden an nur einem Wohnort - veränderte Nachfrage nach professionellen Diensten ergeben.

Eine strategisch-planerische Auseinandersetzung mit den aus multilokalen Alltagsarrangements resultierenden Folgewirkungen für die Bereitstellung und Nutzung von Daseinsvorsorge- und Versorgungsleistungen ist für viele Gemeinden in ländlichen Räumen noch vergleichsweise neu. Dies vor allem, da sich die Folgewirkungen multilokaler Lebensweisen im dörflichen Leben häufig nicht trennscharf von anderen gesellschaftlichen und wirtschaftlichen Entwicklungen vollziehen und von diesen überlagert werden. Für die öffentliche Hand als Leistungserbringerin bzw. rahmensetzende Instanz im Bereich der Daseinsvorsorge und allgemeinen Versorgung verstärken multilokale Lebensweisen dabei Prozesse, die sich durch demografische Entwicklungen, die Veränderung von Haushaltsstrukturen und die sich daraus ergebende veränderte Nachfrage nach Versorgungsleistungen sowie Veränderungen der Arbeitsmärkte und räumlich-zeitlicher Strukturen des Alltagslebens ohnehin vollziehen.

Für Kommunalpolitik und -verwaltung stellt sich - u. a. auch aus kommunalfiskalischen Erwägungen - die Frage nach einem angemessenen Umgang mit Multilokalität. Eine wichtige Frage für Entscheidungsträger*innen adressiert daher die Auswirkungen temporärer An- oder Abwesenheiten auf Kommunalhaushalte und regionale Wirtschaft. Aus strategischer Sicht steht aus dieser Perspektive u. a. die Frage im Raum, ob es sich aus fiskalischen Erwägungen „lohnt“ (vgl. auch Abb. 5.6), 


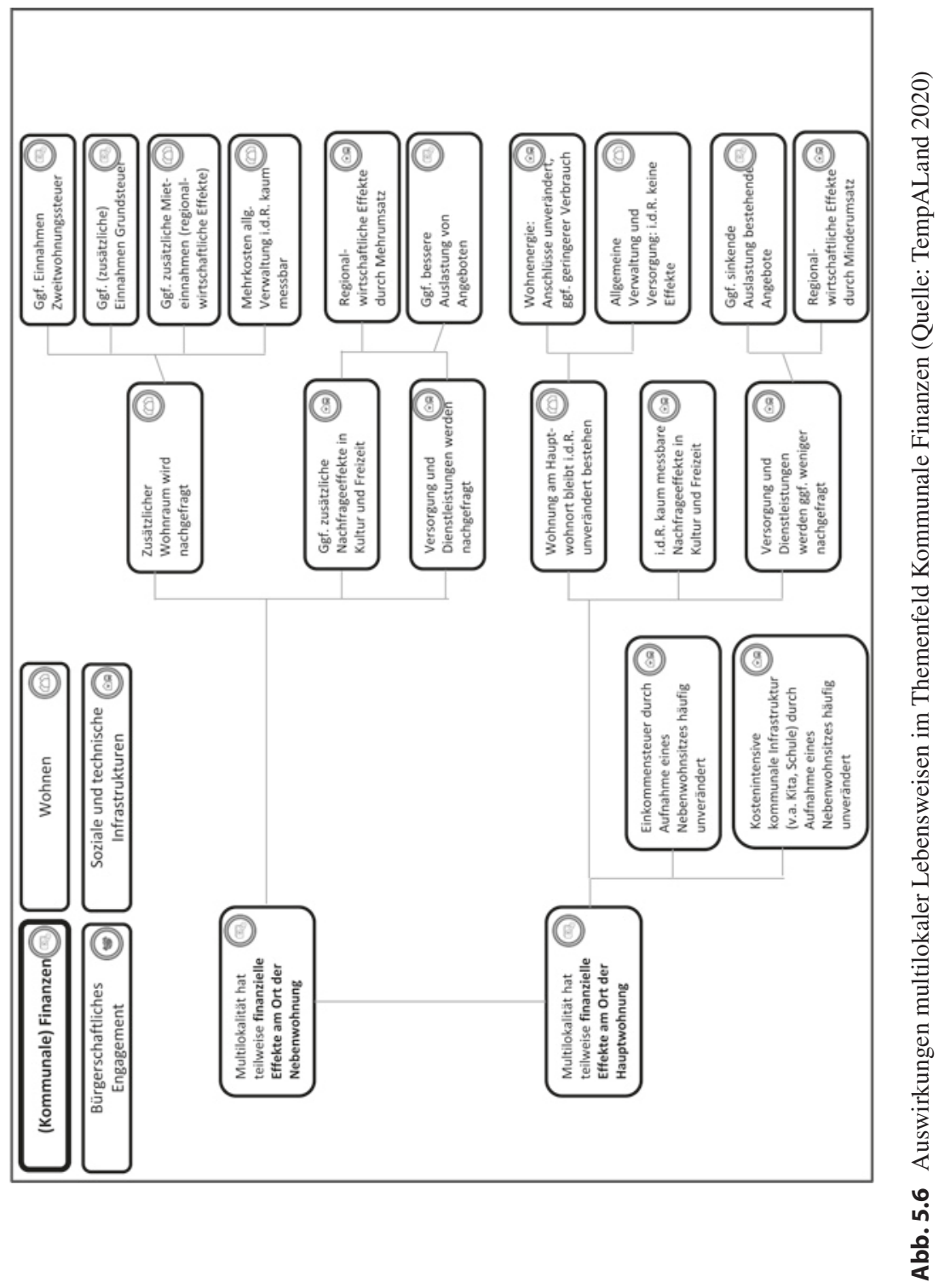


- die Attraktivität von multilokalen Alltagsarrangements (inkl. der An- und Abwesenheiten multilokal lebender Personen) zu stärken,

- multilokal lebende Haushalte zu motivieren, die Erstwohnsitze aller Haushaltsmitglieder in die eigene Gemeinde zu verlegen oder

- die Hauptwohnsitze der Mitglieder multilokal lebender Haushalte in der eigenen Gemeinde zu halten.

Möglichen Überlegungen, welche Handlungsoptionen kommunale Entscheidungsträger*innen im strategischen Umgang mit multilokal lebenden Personen oder Haushalten haben, sollte u. a. eine Bewertung der fiskalischen und regionalwirtschaftlichen Effekte multilokalen Lebens vorgeschaltet werden. Zur Unterstützung dieser Diskussion wurden im Rahmen des Projektes TempALand fiskalische Folgewirkungen multilokaler Lebensweisen für verschiedene Haushaltstypen (im Vergleich zu nicht- oder anders-multilokalen Alltagsarrangements) bilanziert.

\section{Untersuchung von fünf Fallkonstellationen}

Untersucht wurden fünf typische Fallkonstellationen, in denen jeweils eine „Ausgangssituation“ multilokalen Lebens und deren kommunalfiskalische Folgewirkungen mit denen von „Bewertungssituationen“ (= denkbaren Handlungsalternativen der Haushalte) verglichen und bilanziert wurde. Jede Ausgangsbzw. Bewertungssituation bildet einen Untersuchungsfall. Dazu drei Beispiele:

- Ein Mitglied eines Familienhaushaltes arbeitet im Landkreis Diepholz und wohnt in der Nähe des Arbeitsplatzes in einer Zweitwohnung, während der Rest der Familie außerhalb des Landkreises Diepholz dauerhaft am „Hauptwohnort" lebt (Ausgangssituation). Denkbare Handlungsalternativen sind aus Sicht der Familie nun z. B. die Aufgabe der Zweitwohnung im Landkreis Diepholz - etwa weil sich eine berufliche Option in der Nähe des Hauptwohnortes oder ein attraktiveres anderes multilokales Arrangement ergibt (Bewertungssituation 1) oder aber der Umzug der gesamten Familie in den Landkreis Diepholz (Bewertungssituation 2) (Beispiel 1).

- Der Hauptwohnsitz einer Familie ist im Landkreis Diepholz. Ein Familienmitglied arbeitet und wohnt temporär außerhalb des Landkreises (Ausgangssituation). Nun stellt sich Frage, ob es aus Sicht der Kommunen fiskalisch sinnvoll ist, dass die gesamte Familie an den Arbeitsort außerhalb des Landkreises Diepholz zieht (Bewertungssituation 1) oder aber der Zweitwohnsitz außerhalb des Kreises aufgegeben wird und die Familie vollständig im Landkreis Diepholz lebt (Bewertungssituation 2) (Beispiel 2). 
- Ein Haushalt unterhält aus Freizeitgründen einen Zweitwohnsitz im Landkreis Diepholz (Ausgangssituation). Wäre es aus kommunalfiskalischen Erwägungen nun sinnvoll, den Haushalt zu motivieren, den Nebenwohnsitz im Landkreis aufzugeben (Bewertungssituation 1) oder aber seinen Hauptwohnsitz in den Landkreis Diepholz zu verlegen (Bewertungssituation 2) (Beispiel 3)?

Diese (und andere) Fallkonstellationen wurden jeweils für zehn Haushaltstypen betrachtet (z. B. Singlehaushalte, Paarhaushalte, verschiedene Familienhaushalte sowie reine Rentner*innenhaushalte, z. T. unterschieden nach Einkommensniveaus). Insgesamt wurden für jede Gemeinde des Diepholzer Landes 84 Untersuchungsfälle bilanziert.

Für jeden Untersuchungsfall wurde die Höhe unterschiedlicher Einnahme- und Ausgabenarten ermittelt. Dies betrifft auf der Einnahmenseite z. B. Einnahmen aus verschiedenen Steuerarten (z. B. Einkommensteuer, Gewerbe-, Umsatz und ggf. Zweitwohnungssteuer) sowie durch Schlüsselzuweisungen über den kommunalen Finanzausgleich. Auf der Ausgabenseite umfasst die Bilanzierung bspw. Kosten für Kita und Schule sowie die allgemeine Verwaltung. Zusätzlich wurden Abschätzungen der Umsätze im regionalen Einzelhandel sowie Mieteinnahmen durchgeführt und bilanziert.

Jede Fallkonstellation besteht aus mehreren Untersuchungsfällen (jeweils eine Ausgangssituation und mehrere Bewertungssituationen). Im ersten Beispiel wird die Ausgangssituation „Arbeit und Zweitwohnung im Diepholzer Land; Familie woanders" mit zwei Bewertungssituationen verglichen (vgl. Abb. 5.7).

Für die erste Bewertungssituation werden die fiskalischen Effekte untersucht, die entstehen, wenn der/die Arbeitnehmer*in anstelle eines Arbeitsplatzes im Landkreis Diepholz einen Arbeitsplatz außerhalb des Landkreises wählt und der Nebenwohnsitz im Landkreis Diepholz aufgegeben wird. Damit ist ggf. ein Verlust von Einnahmen aus Steuern sowie dem kommunalen Finanzausgleich verbunden. Da die Einkommensteuer ohnehin am Hauptwohnort gezahlt wird, ergeben sich in diesem Beispiel aus der Aufgabe des Zweitwohnsitzes keine (negativen) Folgeeffekte für diese Einkommensart. Für die Samtgemeinde Barnstorf - als Beispiel-Kommune - würden in Bewertungssituation 1 (,geht weg aus Diepholzer Land") in der Summe Mindereinnahmen in Höhe von knapp $90 €$ jährlich entstehen (vgl. Abb. 5.8).

Auf der Ausgabenseite wird unterstellt, dass ein Wegzug eines/r Arbeitnehmers/in keinerlei monetäre Effekte auf die untersuchten Leistungsbereiche der sozialen Infrastruktur sowie der Verwaltung hat. In Bezug auf die Umsätze 


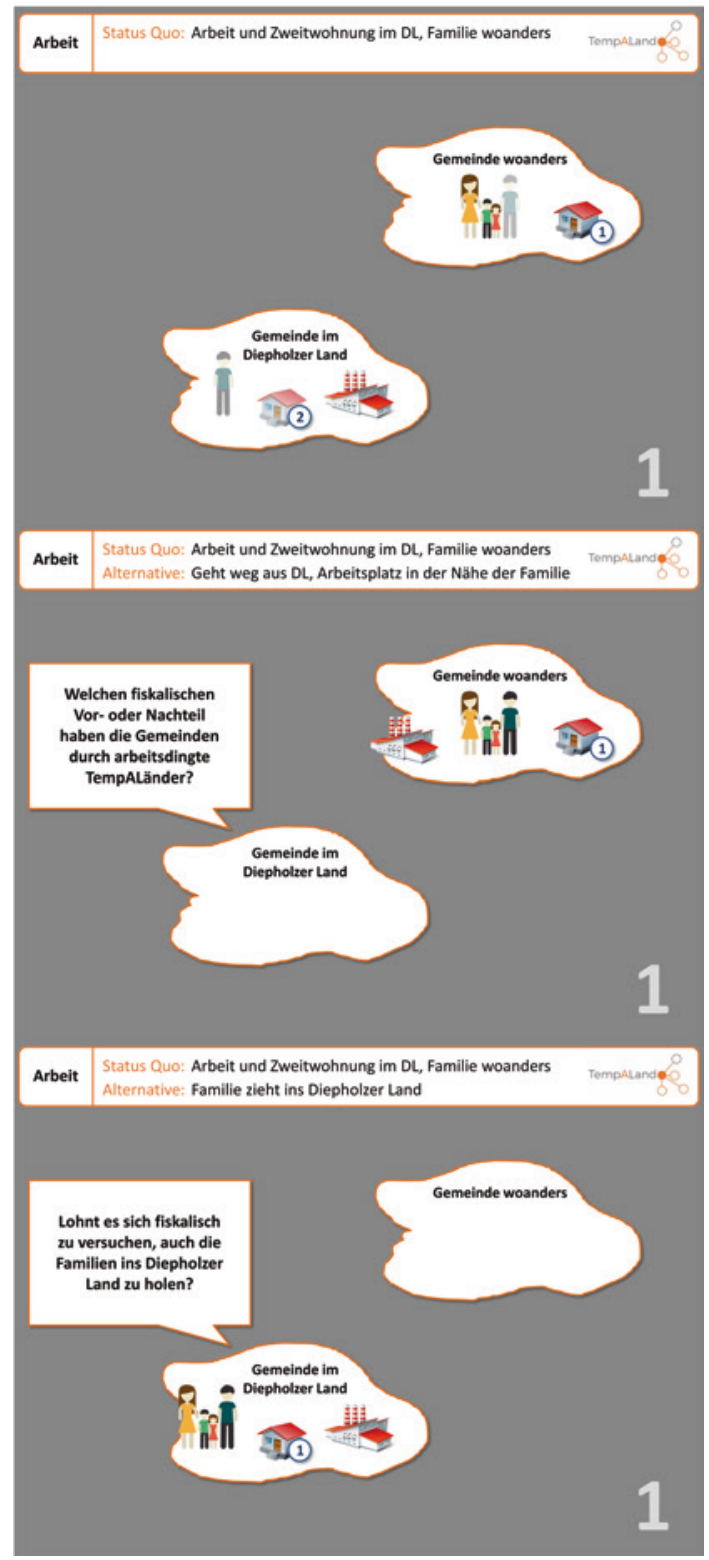

Abb. 5.7 Beispiel: Ausgangssituation und Bewertungssituationen für die Fallkonstellation „Arbeit und Zweitwohnung im Diepholzer Land, Familie woanders“ (Quelle: TempALand 2020) 


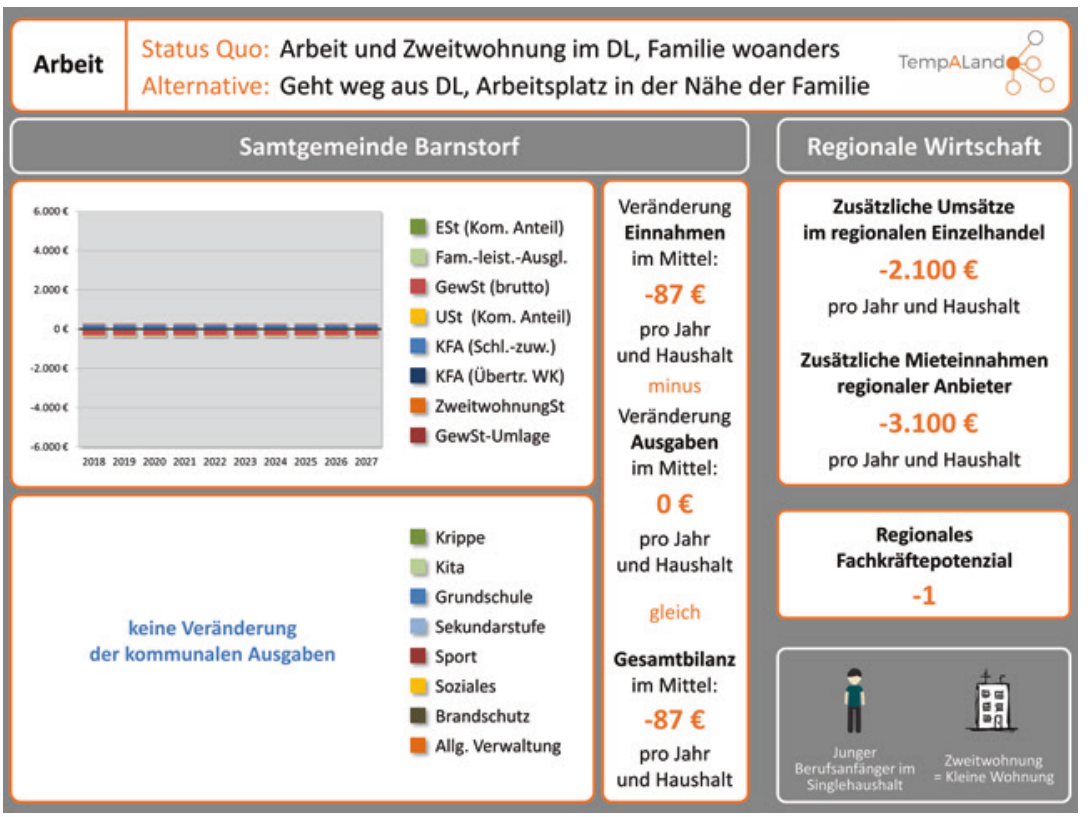

Abb. 5.8 Beispiel 1: Kommunalfiskalische Bilanz der Ausgangssituation „Arbeit und Zweitwohnung im Diepholzer Land, Familie woanders“ und der Bewertungssituation „Geht weg aus DL, Arbeitsplatz in der Nähe der Familie“ für eine Familie mit zwei kleinen Kindern (Quelle: TempALand 2020)

im Einzelhandel sowie die Mieteinnahmen würden sich durch den Fortzug jedoch deutlich negative Folgen ergeben. Der Fortzug temporär anwesender Arbeitskräfte im Landkreis Diepholz wäre in der Gesamtbilanz also mit Einnahmeverlusten verbunden. Diese belaufen sich im hier dargestellten Beispiel auf insgesamt rund $5.300 €$ pro Jahr. Wie Wie Abb. 5.8 zeigt, treffen die Mindereinnahmen hier vor allem privatwirtschaftlich handelnde Akteur*innen (Wohnungsmiete und Einzelhandel). Die Mindereinnahmen für die öffentliche Hand sind demgegenüber eher gering.

Gelingt es hingegen, den gesamten Haushalt zum Umzug in den Landkreis Diepholz zu bewegen (Bewertungssituation 2), würden sich deutlich positive Effekte auf die Einnahmesituation ergeben: Aus dem Zuzug einer Familie mit zwei Erwachsenen und zwei kleinen Kindern resultieren aufgrund der Orientierung an der Anzahl der Personen mit Erstwohnsitz in der Gemeinde spür- 


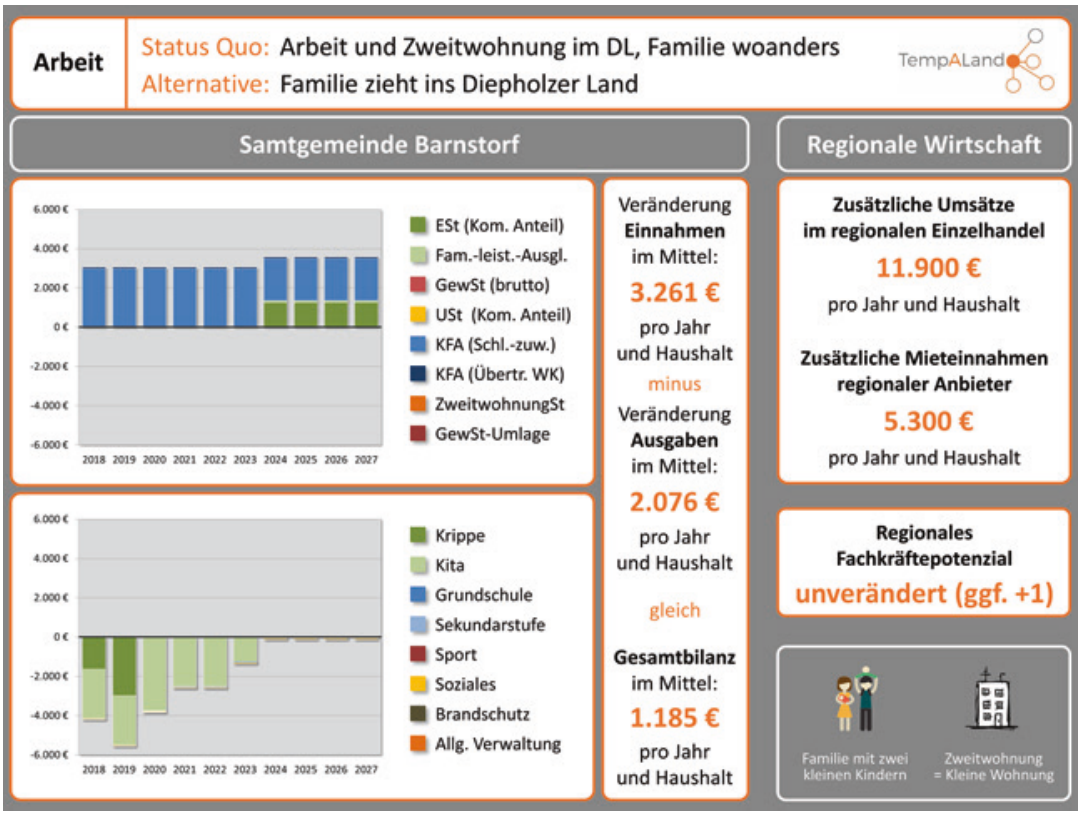

Abb. 5.9 Beispiel 1: Kommunalfiskalische Bilanz der Ausgangssituation „Arbeit und Zweitwohnung im Diepholzer Land, Familie woanders" und der Bewertungssituation „Familie zieht ins Diepholzer Land“ für eine Familie mit zwei kleinen Kindern (Quelle: TempALand 2020)

bare Mehreinnahmen aus dem kommunalen Finanzausgleich sowie - aufgrund der Systematik erst mit einem Zeitverzug von etwa sieben Jahren - auch aus der Einkommensteuer und dem Familienleistungsausgleich im Mittel über 10 Jahre in einer Größenordnung von etwa 3.260 €/Jahr (vgl. Abb. 5.9).

Auf der Ausgabenseite entstehen v. a. durch die kleineren Kinder im Mittel über 10 Jahre Kosten von etwas mehr als $2.000 € / J a h r$. Diese resultieren insbesondere aus kommunalen Aufwendungen zur Sicherstellung der Kindertagesbetreuung. Für alle anderen untersuchten Bereiche auf der Ausgabenseite wird davon ausgegangen, dass die zusätzliche Nachfrage durch den zuziehenden Haushalt eher zu einer Steigerung der Auslastung beiträgt (z. B. Schulen, Sport, kulturelle oder Bildungsinfrastrukturen) und weniger zusätzliche Kosten bzw. 
allenfalls geringe Mehrkosten in den Bereichen Soziales oder allgemeine Verwaltung verursacht ${ }^{1}$.

In der Gesamtbilanz „lohnen“ sich Incomings in diesem Rechenbeispiel aus kommunalfiskalischen Überlegungen also - zumindest gegenüber einem vollständigen Fortzug des Arbeitsnehmers bzw. der Arbeitsnehmerin, d. h. der Aufgabe des multilokalen Alltagsarrangements. Aus kommunalfiskalischer Sicht wäre es aber besonders „lohnenswert“, multilokal lebende Haushalte dazu zu motivieren, den Hauptwohnsitz in den Landkreis Diepholz zu verlegen: Die Bilanz aus kommunalen Einnahmen und Ausgaben verspricht Mehreinnahmen und die zu erwartenden zusätzlichen Umsätze im Einzelhandel sowie durch Mieteinnahmen stärken diese Einschätzung. Sofern aus dem Zuzug resultierende Mehrausgaben im Bereich der Kindertagesbetreuung nicht auftreten (also Zuzug von Single- oder Paarhaushalten bzw. Familienhaushalten mit größeren Kindern) ist die kommunalfiskalische Bilanz noch deutlich positiver.

Beispiel 2 ist die "Spiegelung“ zum ersten Beispiel: Ausgehend von der Situation, dass ein Haushalt mit Erstwohnsitz im Landkreis Diepholz ansässig ist und ein Familienmitglied im Zweitwohnsitz außerhalb des Landkreises lebt, besteht eine Option darin, dass die gesamte Familie aus dem Landkreis Diepholz fortzieht (Bewertungssituation 1). Dies wäre - wie das Beispiel der Stadt Diepholz zeigt - mit geringeren Einnahmen aus Steuern und Zuweisungen verbunden, die auch durch die Einsparungen im Bereich der Ausgaben (insbesondere durch Leistungen der Kindertagesbetreuung) nicht kompensiert werden könnten ( -1.250 €/Jahr). Umsätze im Einzelhandel sowie ggf. Mieteinnahmen wären ebenfalls geringer (vgl. Abb. 5.10) Bei Betrachtung anderer Haushaltstypen, für die sich Einspareffekte im Bereich der Kindertagesbetreuung nicht ergeben würden, wäre die Bilanz aus kommunalfiskalischer Sicht sogar noch ungünstiger.

Erfolgt hingegen eine Verlagerung des Arbeitsplatzes in den Landkreis Diepholz und damit die Aufgabe des multilokalen Alltagsarrangement (Bewertungssituation 2), ergibt sich daraus eine vergleichsweise geringe positive kommunalfiskalische Bilanz, da die wesentlichen Positionen (Einkommensteuer, kommunaler Finanzausgleich, etwaige Kosten für die Kindertagesbetreuung) ohnehin am Hauptwohnsitz verbucht werden.

\footnotetext{
${ }^{1}$ Nicht berücksichtigt sind Sprungkosten, die sich z. B. durch den Ausbau von Kindertagesbetreuungseinrichtungen oder im Bereich der Grundschule ergeben. Die damit verbundenen erheblichen Kostensteigerungen sind jedoch eher Neubauaktivitäten zuzurechnen als Änderungen multilokaler Alltagsarrangements.
} 


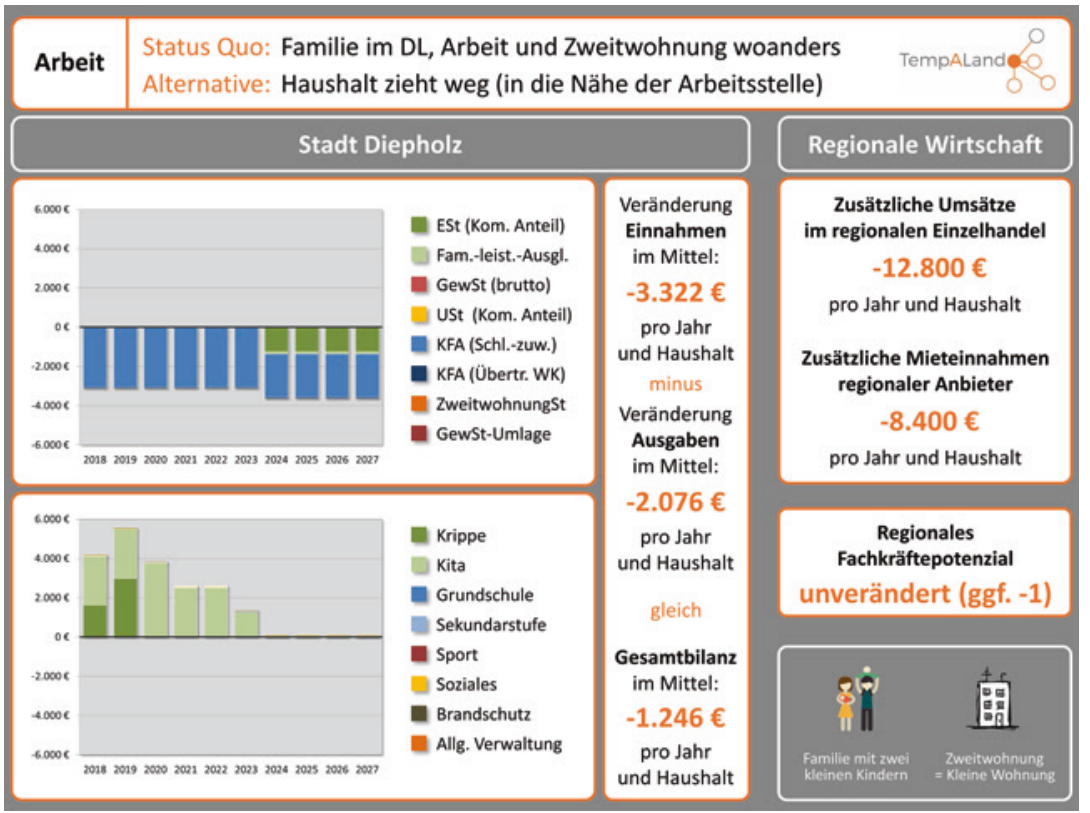

Abb.5.10 Beispiel 2: Kommunalfiskalische Bilanz der Ausgangssituation „Familie im Diepholzer Land, Arbeit und Zweitwohnsitz woanders“ und der Bewertungssituation „Haushalt zieht weg“ für eine Familie mit zwei kleinen Kindern (Quelle: TempALand 2020)

Anhand eines dritten Beispiels sollen Folgewirkungen einer Fallkonstellation im Bereich Freizeit illustriert werden (vgl. Abb. 5.11): Sofern bpsw. ein Rentner*innenehepaar mit höherem Einkommen, das hier aktuell aus Freizeitgründen einen Zweitwohnsitz unterhält, seinen Erstwohnsitz in den Landkreis Diepholz verlegt, lassen sich (geringe) Mehreinnahmen vor allem aus dem kommunalen Finanzausgleich generieren, die die zusätzlichen Aufwendungen übersteigen. Positiv auswirken dürften sich jedoch insbesondere die zusätzlichen Umsätze im regionalen Einzelhandel.

Zusammenfassend lässt sich feststellen, dass sich Multilokalität für die Gemeinden fast immer ,lohnt“. Das heißt einerseits, dass es aus kommunalfiskalischer Perspektive ratsam ist, die Aufgabe eines Zweit- bzw. des Haupt- 


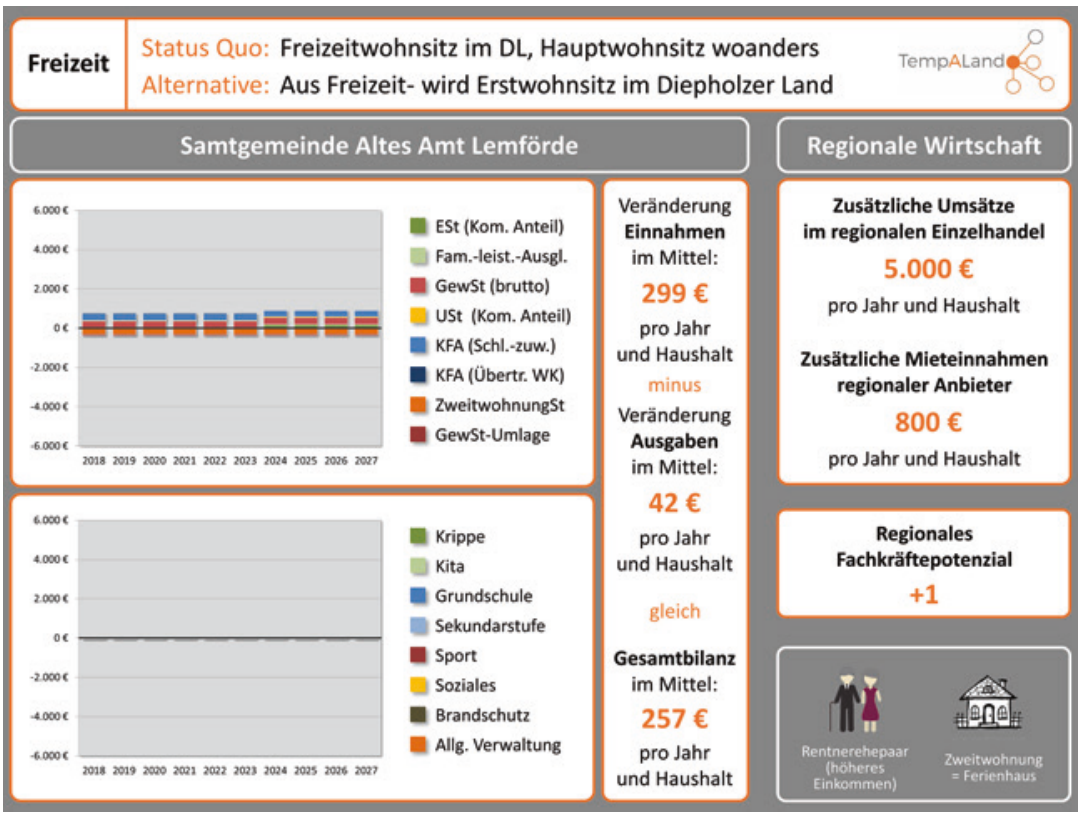

Abb. 5.11 Beispiel 3: Kommunalfiskalische Bilanz der Ausgangssituation „Freizeitwohnsitz im Diepholzer Land, Hauptwohnsitz woanders" und der Bewertungssituation „Aus Freizeit- wird Erstwohnsitz“ für ein Rentnerehepaar mit höherem Einkommen (Quelle: TempALand 2020)

wohnsitzes eines multilokal lebenden Haushaltes im Landkreis Diepholz möglichst zu verhindern („Halten von Multilokalen“). Andererseits zeigt sich, dass auch die nur temporäre Anwesenheit - zumindest im Vergleich zu einem vollständigen Fortzug des Haushaltes - aus Sicht der Kommunen ,lohnenswert“ ist (,Multilokale Lebensweisen ermöglichen“).

Noch deutlich günstigere Effekte ergeben sich dann, wenn es gelingt, multilokal lebende Haushalte zu motivieren, den Erstwohnsitz aller Haushaltsmitglieder in den Landkreis zu verlegen. Die zusätzlichen Einnahmen würden mittel- und langfristig die zu erwartenden Ausgaben immer übersteigen - selbst wenn kurzfristig erhebliche Aufwendungen z. B. im Bereich der Kindertagesbetreuung entstehen würden (,Multilokale zu Bewohner*innen mit Erstwohnsitz machen“). 


\subsection{Multilokale Lebensweisen vor dem Hintergrund einer nachhaltigen Entwicklung}

Die zuvor beschriebenen Auswirkungen multilokaler Lebensweisen in ländlich geprägten Räumen zeigen die vielschichtigen Wirkungen innerhalb der Themenfelder Wohnen, bürgerschaftliches Engagement, kommunale Finanzen und Infrastrukturen. Doch was bedeuten multilokale Lebensweisen vor dem Hintergrund einer nachhaltigen Raumentwicklung? Welche Folgewirkungen sind hier zu erwarten? Mit Blick auf Multilokalität stehen dabei im Folgenden vor allem fünf Aspekte im Vordergrund:

(1) Flächenverbrauch und -neuinanspruchnahme,

(2) Verkehr und CO2-Emissionen,

(3) gesellschaftlicher Zusammenhalt,

(4) (Fach-)Arbeitskräftemangel und

(5) lokale Nachfrage und Wirtschaftskreisläufe.

\section{Flächenverbrauch und -neuinanspruchnahme}

Während der letzten 60 Jahre hat sich die Siedlungs- und Verkehrsfläche in Deutschland mehr als verdoppelt. Nach Angaben des Statistischen Bundesamts ist die Siedlungs- und Verkehrsfläche allein zwischen 1992 und 2016 um 22,2 \% bzw. $8.949 \mathrm{~km}^{2}$ angestiegen (UBA 2018); dies erfolgt oftmals zulasten landwirtschaftlicher Flächen und fruchtbarer Böden. Multilokale Lebensweisen sind dabei - neben dem generellen Trend zu kleineren Haushalten und der steigenden Wohnfläche pro Kopf - mitverantwortlich für den Flächenverbrauch und die Flächenneuinanspruchnahme, indem bspw. Wohnungen am Haupt- und Nebenwohnort bezogen werden. Für diese Entwicklung sind Incomings und Outgoings gleichermaßen verantwortlich: Incomings fragen durch ihr Leben an mehreren Orten zusätzlichen Wohnraum nach und Outgoings geben ihren Wohnraum am Ausgangsort nicht frei, während sie gleichzeitig am anderen Ort zusätzlichen Wohnraum nachfragen. Insbesondere die Nachfrage nach kleinen Wohneinheiten am Nebenort in ländlichen Räumen kann nicht immer erfüllt werden, sodass hier ein Neubaubedarf oftmals zu einer Flächenneuinanspruchnahme führt.

Gleichzeitig ist zu beobachten, dass sich Incomings bei ihren Wohnraumsuchen oftmals auf zentrale, gut erreichbare und arbeitsplatznahe Standorte konzentrieren (z. B. an Bahnhöfen) (Greinke et al. 2018; Greinke et al. 2020). Hier existieren in vielen ländlichen Orten, teilweise auch im Landkreis Diepholz, unzureichend genutzte oder leerstehende Immobilien in den Ortskernen. Damit bestehen einerseits Potenziale zur Nachverdichtung und andererseits zur Nach- 
und Umnutzung alter (zu groß gewordener) Immobilien in kleinere Wohneinheiten. Mit einer geeigneten Innenentwicklungsstrategie können Nach- und Umnutzung trotz des temporären Zuzugs neuer Einwohner*innen durchaus dazu beitragen, die Flächenneuinanspruchnahme in Kommunen nicht zu erhöhen und zu einer nachhaltigen Entwicklung beizutragen.

\section{Verkehr und CO2-Emissionen}

Mobilität ist eine zentrale Voraussetzung für die wirtschaftliche, aber auch gesellschaftliche Entwicklung moderner Industrie- und Dienstleistungsgesellschaften. Allerdings wandeln sich die Mobilitätsanforderungen und -möglichkeiten: Das Mobilitätsverhalten der Menschen wird immer mehr geprägt durch ein „mobil sein (müssen)“, was zu einem erhöhten Verkehrsaufkommen und zu Belastungen für die Umwelt und Gefahren für die menschliche Gesundheit führt.

$\mathrm{Ob}$ und inwiefern Multilokalität bspw. für eine Zunahme von $\mathrm{CO}_{2}$-Emissionen sorgt, kann nicht verlässlich prognostiziert werden, weil (1) die Anzahl multilokal Lebender nicht genau bestimmbar ist (vgl. Beitrag Albrecht/Dittrich-Wesbuer in diesem Band (Kap. 3)), (2) die zurückgelegten Wegstrecken der Multilokalen nicht genau nachvollzogen werden können und (3) die mehrörtigen Lebensweisen sehr individuell und divers sind, wie auch die Distanzen zwischen den jeweiligen Orten. Grundsätzlich ist aber davon auszugehen, dass mehrörtige Lebensweisen für zusätzliches Verkehrsaufkommen sorgen, weil die Multilokalen ihre Orte regelmäßig und abwechselnd aufsuchen. Die erhöhte Mobilität führt i. d. R. zu zunehmenden Verkehr und damit auch zu mehr Emissionen (z. B. $\mathrm{CO}_{2}{ }^{-}$ Emissionen): Insbesondere in ländlichen Räumen werden aufgrund des im Vergleich zu städtischen Räumen schwächer ausgebauten ÖPNV viele Wege mit dem eigenen Pkw zurückgelegt. Gleichzeitig ist jedoch zu beobachten, dass Multilokalität grundsätzlich tägliches Pendeln einschränkt, weil Multilokale zumeist im wöchentlichen Rhythmus zwischen ihren Orten wechseln. So konnte das im Projekt TempALand entwickelte Online-Tool PendlALand aufzeigen, dass tägliches (Fern-)Pendeln häufig deutlich mehr Emissionen verursacht als eine multilokale Lebensweise mit weniger häufigen Ortswechseln (vgl. Beitrag Albrecht/ Dittrich-Wesbuer in diesem Band (Kap. 3); s.a. www.pendlaland.de).

Vor dem Hintergrund einer zunehmenden Digitalisierung taucht hier zusätzlich die Frage auf, ob eine wachsende Technisierung eher zur weiteren Zunahme des Verkehrsaufkommens durch Multilokale oder - aufgrund von digitalen Arbeitsplätzen der sonst „reisenden“ Multilokalen (z. B. zu Hause, im CoWorking Space o.ä.) - zu einer Reduzierung des Verkehrsaufkommens und damit zu einer Trendumkehr führt. Neue Medien und Technisierung werden auch für Multilokale immer wichtiger, d. h. sie nutzen bspw. Messengerdienste und Video- 
technik für den privaten und beruflichen Austausch (Greinke 2020). Zu vermuten ist, dass durch diese digitalen Kontakte eine Abnahme des Verkehrs möglich ist, da Multilokale ihre Pendelrhythmen ausweiten können und dadurch das Verkehrsaufkommen reduziert werden kann.

\section{Gesellschaftlicher Zusammenhalt}

Die Untersuchungen im Landkreis Diepholz zeigen, dass Incomings und Outgoings z. B. aufgrund geringer Zeitverfügbarkeit zumeist ihr freiwilliges Engagement einschränken (müssen). Dadurch ergibt sich die Gefahr, dass das Engagement der Multilokalen ganz wegbricht und z. B. Vereine ,verwaisen". Außerdem sind es vor allem Incomings, die nur selten ein dauerhaftes Engagement anstreben und deshalb die Bindung an den "Zweitort“ i.d. R. eher gering ausgeprägt ist. Für Incomings sind funktionierende Nachbarschaft und soziale Netzwerke am Nebenort (v. a. am Arbeitsort) oftmals zweitrangig, was Auswirkungen auf den örtlichen Zusammenhalt haben kann (u. a. Distanzierung, fehlende Netzwerke etc.). In seltenen Fällen können Incomings aber auch eine Chance für ländliche Räume sein. Denn vereinzelt sind Multilokale durchaus bereit, auch am Nebenort eine Engagementtätigkeit auszuüben. Dadurch bringen sie sich aktiv in (Dorf-)Gemeinschaften mit ihrem Know-how ein und können dafür sorgen, dass Gemeinschaften (nachhaltig) zusammenhalten.

In Bezug auf den sozialen Zusammenhalt zeigt sich im Landkreis Diepholz zudem, dass vor allem Outgoings ihren Wohnort im Landkreis Diepholz als Anker nutzen (Greinke et al. 2020): Eine Engagementtätigkeit am Ausgangsort kann hier ein „Standort-, Bleibe- und Rückkehrfaktor“" sein und stellt v.a. in ländlichen Räumen einen zentralen Baustein für den gesellschaftlichen Zusammenhalt dar. Insbesondere soziale Netzwerke und die Identifikation mit dem Ort oder der Region können dafür sorgen, dass Multilokale sich zugehörig fühlen. Aufgrund der intakten Gemeinschaften am Ausgangsort im Landkreis Diepholz versuchen einige Outgoings, ihr Engagement wenigstens zeitweise aufrecht zu erhalten (z. B. im Sportverein, bei der Freiwilligen Feuerwehr etc.). Zudem sind die zahlreichen Märkte im Landkreis Diepholz Veranstaltungen, zu denen oft Multilokale und Weggezogene phasenweise zurückkehren und sich einbringen. Deutlich wird, dass Multilokalität zwar eine große Herausforderung für den Zusammenhalt und die Dorf- oder Vereinsgemeinschaften darstellt, aber auch Potenziale für die Orte und den Zusammenhalt bedeuten kann (vgl. Beiträge Othengrafen/Greinke/Danielzyk in diesem Band (Kap. 6) und Greinke/Lehmann/ Othengrafen/Seitz in diesem Band (Kap. 7)).

Darüber hinaus haben auch Pendler*innen wenig Zeit zur Verfügung, um sich aktiv zu engagieren. Aus diesem Grund können die zuvor erläuterten Heraus- 
forderungen nicht nur auf multilokal Lebende, sondern auch auf Pendler*innen übertragen werden. Dadurch kann sich die Anzahl der möglichen Engagierten verringern und somit die Risiken in Bezug auf das Engagement und damit zusammenhängend der Erhalt von Daseinsvorsorgeleistungen erschwert werden. Fehlen die Pendler*innen und die Outgoings vor Ort, um sich in Vereinen oder der Feuerwehr zu engagieren, fallen wichtige Aufgaben der Daseinsvorsorge in den ländlichen Räumen brach und können mit der anwesenden Bevölkerung oftmals nur schwer aufrechterhalten werden.

\section{(Fach-)Arbeitskräftemangel}

Der Landkreis Diepholz, insbesondere der ländlich geprägte südliche Teil des Landkreises, ist geprägt durch eine diversifizierte Wirtschaftsstruktur mit mittelständischen und international tätigen Unternehmen. Insbesondere die Automobilzulieferindustrie bildet einen Schwerpunkt im Landkreis. Die Unternehmen im Landkreis Diepholz sind derzeit überwiegend (noch) nicht vom (Fach-)Arbeitskräftemangel betroffen, weil sie ihre Arbeitskräfte zumeist aus der Region gewinnen können. Allerdings sorgen Fusionen und Standortausweitungen oder -neugründungen dafür, dass die Unternehmen im Landkreis zudem häufig mehrere Standorte haben. Das wiederum führt dazu, dass Beschäftigte an die Standorte delegiert werden. Daraus kann eine multilokale Lebensweise entstehen, weil die Unternehmensstandorte so weit auseinander liegen, dass sie mit täglichem Pendeln nicht mehr erreicht werden können. Unternehmen können demnach Treibkräfte für Multilokalität sein, weil sie Beschäftigte in diese Lebensweise „drängen“ (Greinke 2020).

Darüber hinaus gibt es bereits Unternehmen im Landkreis Diepholz, die vom (Fach-)Arbeitskräftemangel betroffen sind, weil sie keine qualifizierten Beschäftigten im näheren Umfeld finden, z. B. aufgrund spezialisierter Tätigkeiten (Greinke 2020). Dementsprechend beschäftigen sie bereits Multilokale, die sich nicht vorstellen können, komplett in den Landkreis Diepholz umzuziehen, weil ihr Ausgangsort außerhalb des Landkreises liegt und sie diesen nicht aufgeben möchten.

Aber nicht nur im Landkreis Diepholz wird zunehmender Fachkräftemangel zu einer Herausforderung. Incomings können folglich eine Chance sein, den Fachkräftemangel in den Unternehmen einzudämmen (Greinke et al. 2018, Greinke/ Hilti 2019), indem sie zumindest zeitweise Arbeitsplätze besetzen und somit ggf. den Fortbestand von Betrieben sicherstellen. Ein „Bleiben“ der Bevölkerung und der Multilokalen, also sesshaft sein oder werden, kann damit einen Beitrag zur nachhaltigen Regionalentwicklung in ländlichen Räumen leisten. 


\section{Lokale Nachfrage und Wirtschaftskreisläufe}

Ökonomisch betrachtet können insbesondere die freizeitlich genutzten Unterkünfte, z. B. in Ferienhausgebieten, für einen finanziellen Mehraufwand bei den Kommunen sorgen. Hier müssen zwar keine neuen Infrastrukturen (Straßen, Verund Entsorgungsinfrastrukturen etc.) geschaffen, aber dennoch dauerhaft unterhalten werden, auch wenn die freizeitbedingten Incomings ihre Unterkünfte am Nebenort oft nur saisonal bewohnen. Multilokalität bedeutet aber nicht zwingend, dass für die Kommunen - z. B. durch Erschließung von Wohnraum oder Infrastrukturen zusätzliche Kosten entstehen.

Multilokalität kann sich für Kommunen sowohl aus kommunalfiskalischer Perspektive („Halten von Multilokalen“) als auch anstelle eines vollständigen Fortzugs des Haushaltes („Multilokale Lebensweisen ermöglichen“) lohnen, indem sie bspw. durch steuerliche Zuweisungen (z. B. Zweitwohnungssteuer in Ferienhausgebieten) Einnahmen generieren, die jedoch auch zweckgebunden auszugeben sind. Kommunen und Wirtschaft können aber vor allem aufgrund zusätzlicher Kaufkraft, die die multilokalen Incomings in den Landkreis Diepholz bringen (bspw. Einzelhandel und Dienstleitungen), profitieren (vgl. Beitrag Greinke/Lange in diesem Band (Kap.4); vgl. Dittrich-Wesbuer 2016: 49; DittrichWesbuer et al. 2014: 367; Leubert 2013: 140; Weichhart/Rumpolt 2015: 53). Zudem können Outgoings dafür sorgen, dass durch ihre Einkäufe am Ausgangsort Kaufkraft aufrechterhalten bleibt.

\section{Fazit}

Die Wirkungen von Multilokalität in ländlichen Räumen innerhalb der Themenfelder Wohnen, bürgerschaftliches Engagement, kommunale Finanzen und Infrastrukturen sind sehr vielschichtig. Daraus ergeben sich komplexe Folgewirkungen für eine nachhaltige Raumentwicklung, aus denen sowohl Chancen als auch Herausforderungen für die Kommunen, die Wirtschaft und die Bevölkerung resultieren können. In vielen Kontexten kann es sinnvoll sein, Multilokale als Zuziehende zu gewinnen oder zumindest die mehrörtigen Arrangements aufrechtzuerhalten (z. B. um (Fach-)Arbeitskräftemangel einzudämmen). Die Auswirkungen von Multilokalität sind bislang in kommunalpolitischen Debatten kein zentrales Thema, weil u. a. quantitative Aussagen zu Multilokalen fehlen oder zu gering sind. Allerdings zeigt dieser Beitrag, dass Multilokalität dafür sorgen kann, dass sich insgesamt Prozesse verstärken, die die kommunale Ebene schon heute vor erhebliche Herausforderungen stellen. Daher könnte und sollte Multilokalität zukünftig ein wichtiges Handlungsfeld für die räumliche Planung, Politik und Verwaltung sowie Unternehmen und in der Zivilgesellschaft sein. 


\section{Literatur}

Aring, J. (2010): Gleichwertige Lebensverhältnisse - Inverse frontiers - Selbstverantwortungsräume. In: Oswalt, P. (Hg.): Weniger ist Zukunft. 19 Städte - 19 Themen: Internationale Bauausstellung Stadtumbau Sachsen-Anhalt 2010 (Katalog zur gleichnamigen Ausstellung in der Stiftung Bauhaus Dessau, 9. April 2010 bis 16. Oktober 2010). Berlin: Jovis, 764-777.

Becker, E./Gualini, E./Runkel, C./Strachwitz Graf, R. (Hg.) (2010): Stadtentwicklung, Zivilgesellschaft und bürgerschaftliches Engagement. Stuttgart.

Bieker, S./Knieling, J./Sinning, H./Othengrafen, F. (2004): STADT+UM+LAND 2030 Region Braunschweig: Kooperative Stadt-Region; Stadt 2030 Forschungsergebnisse, Braunschweig.

Blotevogel, H.-H./Danielzyk, R. (2006): Ungleichwertigkeit der Lebensverhältnisse Herausforderung für die Raumordnungspolitik? In: Selle, K. (Hg.): Praxis der Stadt- und Regionalentwicklung. Analysen. Erfahrungen. Folgerungen. Dortmund: Rohn, 59-71.

BMFSFJ (2016): Zweiter Engagementbericht 2016. Demografischer Wandel und Bürgerschaftliches Engagement: Der Beitrag des Engagements zur lokalen Entwicklung. Berlin.

Deutscher Bundestag (2002): Bericht der Enquete-Kommission ,Zukunft des Bürgerschaftlichen Engagements“. Bürgerschaftliches Engagement: auf dem Weg in eine zukunftsfähige Bürgergesellschaft. Drucksache 14/8900.

Dirksmeier, P. (2010): Multilokalität als Abwesenheit: eine Herausforderung für landschaftliche attraktive ländliche Räume - das Beispiel Tegernsee/Stadt. Europa Regional 10/2010 (2-3), 60-70.

Dittrich-Wesbuer, A./Eichhorn, S./Tippel, C. (2014): Relevanz multilokaler Phänomene in der Stadtentwicklung. Ergebnisse aus Expertenbefragungen in Nordrhein-Westfalen. In: Hamman, P./Blanc, M./Duchêne-Lacroix, C./Freytag, T./Kramer, C. (Hg.) (2014): Questionner les mobilités résidentielles à l'aune de la multilocalité. Strasbourg: Néothèque Éditions, 275-357.

Dittrich-Wesbuer, A./Föbker, S./Sturm, G. (2015): Multilokales Wohnen: Empirische Befunde zur Verbreitung in Deutschland. In: Weichhart, P./Rumpolt P. A. (Hg.): Mobil und doppelt sesshaft. Studien zur residenziellen Multilokalität. Wien, Abhandlungen zur Geographie und Regionalforschung, Nr.18, 121-143.

Dittrich-Wesbuer, A. (2016): Multilokalität und Stadtentwicklung. Veränderte Muster räumlicher Mobilität und ihre lokalen Implikationen. Genehmigte Dissertation. Hannover: Selbstverlag.

Dittrich-Wesbuer, A./Sturm, G. (2020): Quantitative Daten I: Amtliche Daten zur Verbreitung multilokaler Lebensformen in Deutschland, Österreich und der Schweiz. In: Danielzyk, R./ Dittrich-Wesbuer, A./Hilti, N./Tippel, C. (Hg.) (2020): Multilokale Lebensführungen und räumliche Entwicklungen - ein Kompendium. Hannover. Forschungsberichte der ARL 13. URN : https://nbn-resolving.de/urn:nbn:de:0156-09764, 104-111.

Greinke, L./Lange, L./Othengrafen, F. (2018): Multilokales Wohnen in ländlichen Räumen - Chancen und Herausforderungen. PlanerIn 18 (3), 44-46.

Greinke, L./Lange, L./Othengrafen, F. (2020): Auswirkungen temporärer An- und Abwesenheiten auf den ländlich geprägten Landkreis Diepholz (Forschungsprojekt TempALand). In: Danielzyk, R./Dittrich-Wesbuer, A./Hilti, N./Tippel, C. (Hg.): Multilokale Lebens- 
führungen und räumliche Entwicklungen - ein Kompendium. Hannover. = Forschungsberichte der ARL 13, 337-343.

Greinke, L./Hilti, N. (2019): Temporär genutzte Räume von berufsbedingt multilokal Lebenden. Herausforderungen und Potenziale für betriebliche und regionale Entwicklungen in ländlichen Räumen Niedersachsens. Raumforschung und Raumordnung 78 (1): 1-17.

Greinke, L. (2020): Berufsbedingte Multilokalität in ländlichen Räumen Niedersachsens. Gesellschaftliche und räumliche Auswirkungen als planerische Herausforderung am Beispiel des Landkreises Diepholz. Ländliche Räume: Beiträge zur lokalen und regionalen Entwicklung Bd. 7, LIT-Verlag Berlin.

Henkel, Gerhard (2010): Stärken und Schwächen unserer Dörfer - Wie könnte ein Fitnessprogramm für die Zukunft aussehen? Flächenmanagement und Bodenordnung, 2, 54-61.

InWIS Forschung/Beratung GmbH (InWIS) (2016): Wohnraumversorgungskonzept Landkreis Diepholz, Bochum.

Kahle, I./Schäfer, D. (2005): Ehrenamt und bürgerschaftliches Engagement. Ergebnisse der Zeitbudgeterhebung 2001/2002. Wirtschaft und Statistik, 4, 311-317.

Kötter, T. (2009): Innenentwicklung der Dörfer und Revitalisierung der Dorfkerne. Von der Dorferneuerung zum Dorfumbau. In: Vorstand der Deutschen Landeskulturgesellschaft (Hg.): Dörfer ohne Menschen. Zwischen Abriss, Umnutzung und Vitalisierung. Müncheberg: Eigenverlag (Schriftenreihe Deutsche Landeskulturgesellschaft, Sonderheft 02), 6-27.

Lange, L. (2018): Leben an mehreren Orten. Multilokalität und bürgerschaftliches Engagement in ländlich geprägten Räumen Niedersachsens. Berlin.

Leubert, N. (2013): Heute hier. Morgen dort. Chancen und Herausforderungen multilokaler Lebensstile in Wolfsburg. Graue Reihe des Instituts für Stadt- und Regionalplanung. Band 47. Berlin: Universitätsverlag der TU Berlin.

Landesamt für Statistik Niedersachsen (LSN) (2019): LSN-Online - Regionaldatenbank. Aufgerufen am 13.06.2019, URL: https://www1.nls.niedersachsen.de/statistik/html/ default.asp.

NBank (Hg.) (2017): Wohnungsmarktbeobachtung 2016/2017. Bezahlbares Wohnen für alle. Perspektiven für Niedersachsen 2015-2035, Hannover.

NBank (Hg.) (2019): Wohnungsmarktbeobachtung 2019. Wohnungsmarktbeobachtung 2019. Zukunftsfähige Wohnungsmärkte Perspektiven für Niedersachsen bis 2040, Hannover.

Olk, T./Hartnuß, B. (2011): Bürgerschaftliches Engagement. In: Olk, T./Hartnuß, B. (Hg.): Handbuch Bürgerschaftliches Engagement. 1. Aufl., 145-161.

Prognos AG; AMB Generali Holding AG; Generali Zukunftsfonds (Hg.) (2008): Engagementatlas 2009. Aachen.

Rüger, H./Becker, K. (2011): Berufsmobilität, Geschlecht und Lebensform. Berufsbedingte räumliche Mobilität und die Folgen für die Vereinbarkeit von Familie und Beruf und partnerschaftliche Arbeitsteilung. In: Klammer, U./Motz, M. (Hg.): Neue Wege gleiche Chancen. Stellungnahmen zum Ersten Gleichstellungsbericht der Bundesregierung. Wiesbaden, 363-396.

Schier, M. (2014): Multilokalität von Familie in Deutschland. Geographische Rundschau. 66, 10-17. 
Siedentop, S./Junesch, R./Uphues, N./Schöfl, G. (2011): Der Beitrag der ländlichen Räume Baden-Württembergs zu wirtschaftlicher Wettbewerbsfähigkeit und sozialer Kohäsion. Positionsbestimmung und Zukunftsszenarien. Forschungsvorhaben im Auftrag des Ministeriums für Ländlichen Raum und Verbraucherschutz Baden-Württemberg (Abschlussbericht). Stuttgart.

Steinführer, A. (2015): Bürger in der Verantwortung. Veränderte Akteursrollen in der Bereitstellung ländlicher Daseinsvorsorge. Raumforschung und Raumordnung, 73(1), $5-16$.

Tippel, C. (2020): Berufsbedingt multilokale Lebensführung: Begrifflichkeiten, Entstehungskontexte und räumliche Auswirkungen. In: Danielzyk, R./Dittrich-Wesbuer, A./Hilti, N./Tippel, C. (Hg.) (2020): Multilokale Lebensführungen und räumliche Entwicklungen - ein Kompendium. Hannover. Forschungsberichte der ARL 13. URN : https://nbn-resolving.de/urn:nbn:de:0156-09764, 91-97.

Voß, W./Güldenberg, E./Jürgens, A./Kirsch-Stracke, R./Streibel, N. (2011): Modellprojekt Umnutzung landwirtschaftlicher Altgebäude und Hofanlagen als Beitrag zur Vitalisierung der Ortskerne. Abschlussbericht der wissenschaftlichen Begleitforschung. Hannover.

Weichhart, P. (2009): Multilokalität - Konzepte, Theoriebezüge und Forschungsfragen. Informationen zur Raumentwicklung ,1/2.2009, 1-14.

Weichhart, P./Rumpolt P. A. (Hg.) (2015): Mobil und doppelt sesshaft. Studien zur residenziellen Multilokalität. Wien, Abhandlungen zur Geographie und Regionalforschung, Nr.18, 121-143.

Zensusdatenbank (2019): Zensus 2011 der Statistischen Ämter des Bundes und der Länder. Aufgerufen am 13.06.2019, URL: www.statistik.niedersachsen.de.

Open Access Dieses Kapitel wird unter der Creative Commons Namensnennung 4.0 International Lizenz (http://creativecommons.org/licenses/by/4.0/deed.de) veröffentlicht, welche die Nutzung, Vervielfältigung, Bearbeitung, Verbreitung und Wiedergabe in jeglichem Medium und Format erlaubt, sofern Sie den/die ursprünglichen Autor(en) und die Quelle ordnungsgemäß nennen, einen Link zur Creative Commons Lizenz beifügen und angeben, ob Änderungen vorgenommen wurden.

Die in diesem Kapitel enthaltenen Bilder und sonstiges Drittmaterial unterliegen ebenfalls der genannten Creative Commons Lizenz, sofern sich aus der Abbildungslegende nichts anderes ergibt. Sofern das betreffende Material nicht unter der genannten Creative Commons Lizenz steht und die betreffende Handlung nicht nach gesetzlichen Vorschriften erlaubt ist, ist für die oben aufgeführten Weiterverwendungen des Materials die Einwilligung des jeweiligen Rechteinhabers einzuholen.

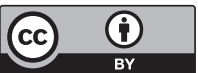

